\title{
Quasi-static crack propagation in plane and plate structures using set-valued traction-separation laws
}

\begin{tabular}{|r|l|}
\hline Journal: & International Journal for Numerical Methods in Engineering \\
\hline Manuscript ID: & NME-Sep-06-0559.R2 \\
\hline Wiley - Manuscript type: & Research Article \\
\hline Author: & 19-Jul-2007 \\
\hline Complete List of Authors: & $\begin{array}{l}\text { Areias, Pedro Miguel de Almeida; University of Illinois at Urbana- } \\
\text { Champaign, Computational Science and Engineering } \\
\text { Rabczuk, Timon; University of Canterbury, Department of } \\
\text { Mechanical Engineering }\end{array}$ \\
\hline Keywords: & $\begin{array}{l}\text { Crack propagation, Cohesive traction-separation, Complementarity, } \\
\text { Plane problems, Plates }\end{array}$ \\
\hline
\end{tabular}

\section{(5) ScholaroNE \\ Manuscript Central}


INTERNATIONAL JOURNAL FOR NUMERICAL METHODS IN ENGINEERING

Int. J. Numer. Meth. Engng 2004; 00:1-6 Prepared using nmeauth.cls [Version: 2002/09/18 v2.02]

\section{Quasi-static crack propagation in plane and plate structures using set-valued traction-separation laws}

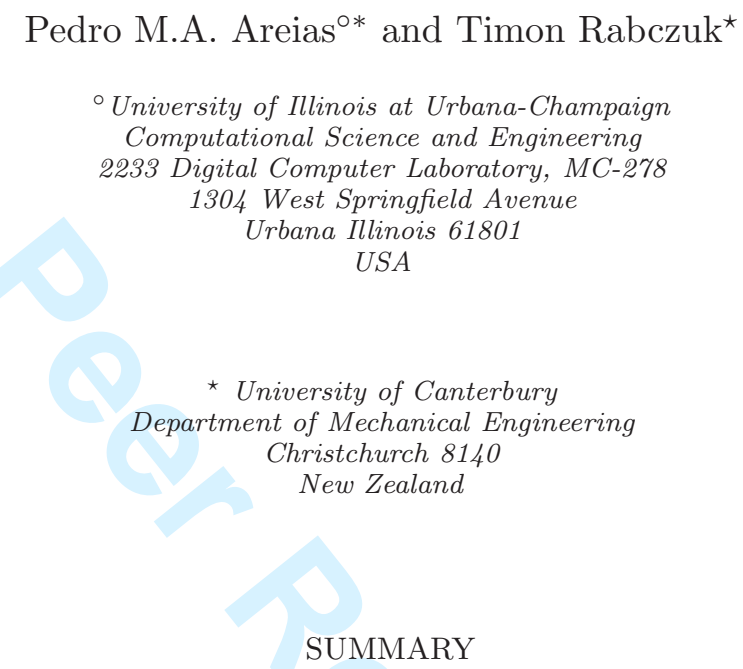

We introduce a numerical technique to model set-valued traction-separation laws in plate bending and also plane crack propagation problems. By use of recent developments in thin (Kirchhoff-Love) shell models and the extended finite element method, a complete and accurate algorithm for the cohesive law is presented and is used to determine the crack path. The cohesive law includes softening and unloading to origin, adhesion and contact. Pure debonding and contact are obtained as particular (degenerate) cases. A smooth root finding algorithm (based on the trust region method) is adopted. A step-driven algorithm is described with a smoothed law which can be made arbitrarily close to the exact non smooth law. In the examples shown the results were found to be step-size insensitive and accurate. In addition, the method provides the crack advance law, extracted from the cohesive law and the absence of stress singularity at the tip.

KEY WORDS: Crack propagation, cohesive traction-separation law, complementarity, plane problems, plates

\section{INTRODUCTION}

Cohesive models of fracture are accepted as representative of the near-tip nonlinear zone of quasi-brittle and certain ductile materials. The idea is attributed to Barenblatt [1]. These

* Correspondence to: Pedro M.A. Areias, University of Illinois at Urbana-Champaign; E-mail address: pareias@uiuc.edu

Copyright (C) 2004 John Wiley \& Sons, Ltd. 
models have certain attractive properties; for example, a pre-existent crack is not necessary. Neither is the stress singularity in the crack tip which requires a specific discretization to be represented. Because of this, numerous applications were carried out by several authors (reviews are available in $[2,3,4,5]$ ) and some software packages can currently be used. However, it is known that for the problem to be well-posed, cohesive models must include an initially rigid behavior (or at least as stiff as possible, as indicated by Alfano and Crisfield [6]). In finite element codes, initially rigid models (also called extrinsic, see [7]) cannot be implemented when only displacement degrees of freedom are used, and regularization adversely affects the results $[7,8]$. In particular, the crack profile and the cohesive zone length result different.

For explicit codes, Papoulia et al. [9] and Sam et al. [8] proposed specialized time-step solutions, but the results can suffer from interpenetration stress locking due to opposite signs in stress and opening displacement (as indicated by the conclusions of Papoulia et al. [9]) and a new model was said to be required. At least two algorithms of solution to deal with the initially rigid behavior exist: event-driven and step-driven algorithms. A generic comparison between these methods is provided by Leine and Nijmeijer [10]. In summary, event driven algorithms are analogous to constraint active-set strategies in optimization problems whereas step-driven methods use the state of the constraints at each pre-established time-step. Step-driven methods are appropriate for problems with a large number of complementarity conditions, but are usually less accurate in terms of resolution [10]. We make use of a fixed-step driven method and a smooth root-finder.

The locking effect did not occur in the examples shown here. If we identify the problem as a NCP (non-linear complementarity problem, see also [11]), adhesion and unloading share the same law unrelated with the behavior of the adjacent continuum. We can therefore employ a rigorous surface-based traction-separation law with any particular continuum problem, regardless of the method used to obtain the stress in the continuum. The advantages of this approach were pointed out by T. Belytschko to the authors of this manuscript [12].

This differs from some perspectives in this field, where a continuum-based cohesive law (the continuum constitutive law has to correctly represent the pre-localization response, the localization surface and, in addition, the post-localization response) is often proposed. In the work of Huespe et al. [13] the authors claim that no discontinuities occur with the classical approach, but a comparison was not provided there. Here, we perform tests where interpenetration stress locking is absent. The stress state can travel in a vertical half-line limited by the maximum stress.

Analytical and numerical studies of fracture in plain strain and thin plates (plane stress) have been disseminated in the literature (see, e.g. $[14,15])$. These fail to provide a comprehensive solution to the traction-separation behavior.

The introduction of set-valued traction-separation laws in the discrete nonlinear problem of cohesive fracture leads to a NCP that can be solved by B-smooth solvers [16], interior point methods or smoothing techniques [17]. The traction-separation laws contain an infinite initial stiffness and also a compliance in loading which varies with time as an internal variable evolves (note that, according to Elices et al. [4], hardening should be absent). Smoothing methods can be implemented and use made of Newton method for smooth functions if some modifications are introduced in the discrete formulation.

We shall see that if these are done, both smooth and non-smooth problems can be solved with the same framework. From a solution viewpoint, either line-search or trust region methods can be used in the inner stage of the method; a continuation method with adaptive smoothing

Int. J. Numer. Meth. Engng 2004; 00:1-6 
parameters was proposed by Chen and Harker [18].

Trust-region methods are appropriate for continuous but not necessarily "quadratic-like" functions: the search direction is obtained as a convex combination of Newton and the steepest descent Cauchy step. A quadratic merit function is the indicator for the coefficient of the combination.

Caution is exerted in the formulation of the modified cohesive problem, as smoothing can introduce artificial spikes in the cohesive law. Details about this are given in section 3. In addition, only quasi-static growth is shown in this paper and the crack propagation is known to be stable for the experimental beam tests shown.

The paper is organized as follows: in section 2 the relevant quantities and the formulation are introduced; in section 3 the mixed method is discussed with a bi-linear cohesive law. Section 4 describes the crack advance condition, which is based on the tip force. Both $2 D$ and plate bending examples are presented in section 5 . Finally, the conclusion follows in section 6 .

\section{FORMULATION AND DISCRETIZATION}

The constrained quasi-static thin plate bending problem consists of equilibrium equation, mass conservation principle, the Kirchhoff-Love constraint, Dirichlet and Neumann boundary conditions, stress-displacement jump cohesive law and the Cauchy Lemma for the cohesive surface. These are grouped in the equation system (1). Figure 1 depicts the relevant ingredients. A $2 D$ plane stress (or strain) problem can be solved with the same underlying theory by prescribing the out-of-plane displacement and modifying the elastic law.

The relevant domains are: the body in deformed $\Omega$ and reference $\Omega_{0}$ configurations, the crack core in the deformed $\Gamma_{c}$ and reference $\Gamma_{c 0}$ configurations, the Dirichlet boundary $\Gamma_{\bar{u}}$ and Neumann boundary $\Gamma_{\bar{t}}$.

$$
\begin{aligned}
& \left.\begin{array}{c}
\operatorname{Div} \boldsymbol{P}^{T}+\boldsymbol{b}_{0}=0 \\
\rho_{0}=\operatorname{det}[\boldsymbol{F}] \rho \\
C_{i 3}=\delta_{i 3}
\end{array}\right\} \quad \text { for } \quad \boldsymbol{x} \in \Omega \\
& \boldsymbol{u}=\overline{\boldsymbol{u}} \text { for } \boldsymbol{x} \in \Gamma_{\bar{u}} \\
& \boldsymbol{t}=\overline{\boldsymbol{t}} \quad \text { for } \quad \boldsymbol{x} \in \Gamma_{\bar{t}} \\
& e q\left(\sigma_{n}, \Delta u\right)=0 \quad \text { for } \quad \boldsymbol{x} \in \Gamma_{c} \\
& \operatorname{det}[\boldsymbol{F}] \sigma_{n}=\boldsymbol{n}_{c}^{T}\left(\boldsymbol{P} \boldsymbol{N}_{c}\right) \quad \text { for } \quad \boldsymbol{x} \in \Gamma_{c}
\end{aligned}
$$

In (1), $\boldsymbol{P}$ is the first Piola-Kirchhoff stress tensor, $\boldsymbol{b}_{0}$ is the volume force vector in the reference configuration; $\rho_{0}$ and $\rho$ are the reference and deformed mass density, respectively. The customary notation is employed for the coordinates of a given point $X: \boldsymbol{X}$ is the reference position vector and $\boldsymbol{x}$ is the deformed reference position vector. The deformation gradient, $\boldsymbol{F}$, is given by $\frac{\partial \boldsymbol{x}}{\partial \boldsymbol{X}}$. The deformed and reference cohesive surface normals are related by:

$$
\boldsymbol{N}_{c}=\left(\frac{\partial \boldsymbol{x}}{\partial \boldsymbol{X}}\right)^{T} \boldsymbol{n}_{c}
$$

Note that (2) could also be written, in a more traditional form, with the use of norms. 
The outer boundary of $\Omega, \partial \Omega$ is partitioned into two boundaries: $\partial \Omega=\Gamma_{\bar{u}} \cup \Gamma_{\bar{t}}$. For a plate or shell, mid surface coordinates $\theta^{1}$ and $\theta^{2}$ and a signed distance to the mid surface $\theta^{3}$ are introduced so that an appropriate form of the equilibrium equation is obtained, based on oneto-one correspondence $\boldsymbol{X} \leftrightarrow \theta^{i}$. For convenience, the mid surface coordinates $\theta^{1}$ and $\theta^{2}$ are indexed as $\alpha$ and $\beta$ (see [19]). Derivatives of a quantity $(\bullet)$ with respect to $\alpha$ are represented as $(\bullet)_{,}$.

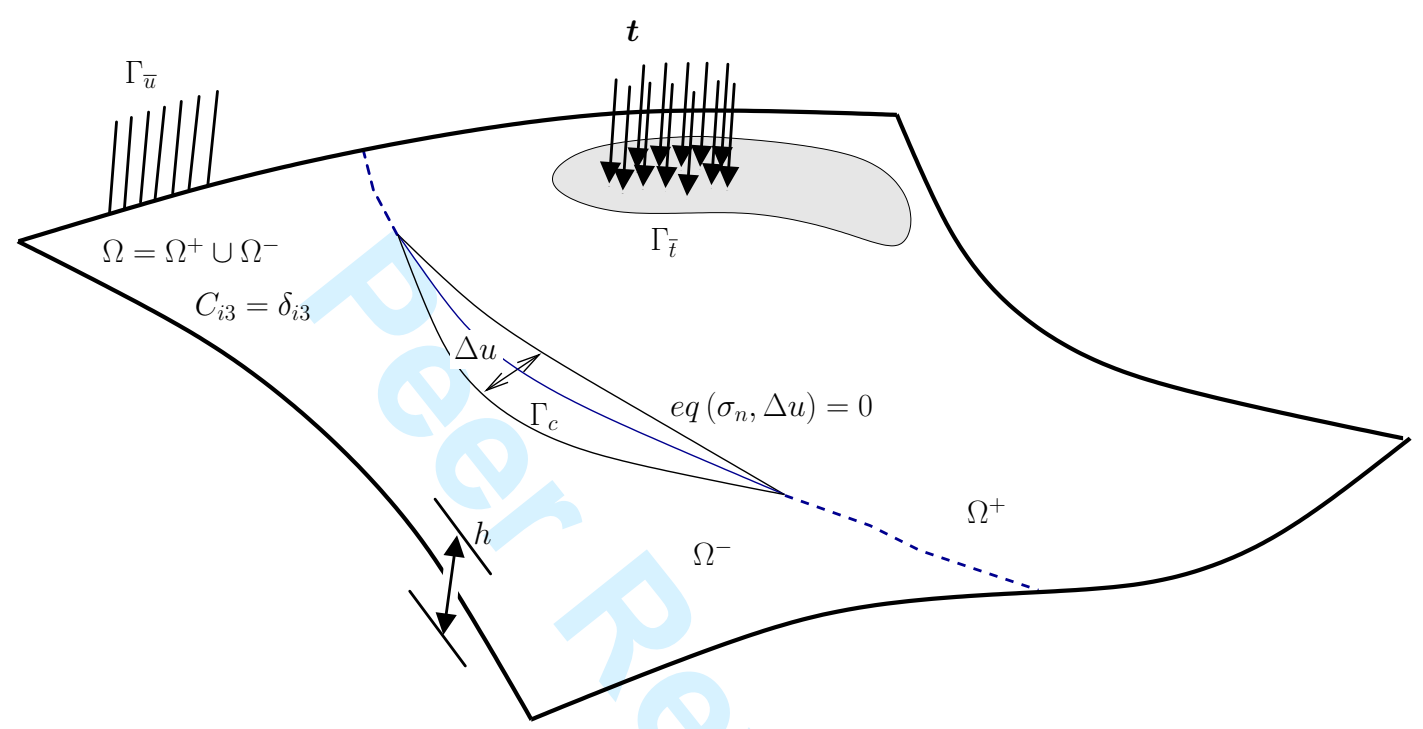

Figure 1. Relevant quantities of the cohesive problem. The equation $e q\left(\sigma_{n}, \Delta u\right)=0$ represents the force-displacement constraint corresponding to a particular traction-separation model. The thickness $h$ is assumed to be uniform.

Using this nomenclature, the weak form of the equilibrium equation is:

$$
\int_{\Omega} s^{\alpha \beta} \boldsymbol{x}_{, \beta} \cdot \delta \boldsymbol{x}_{, \alpha} \sqrt{G} \mathrm{~d} \theta^{1} \mathrm{~d} \theta^{2} \mathrm{~d} \theta^{3}=\delta W_{E}
$$

where $s^{\alpha \beta}$ are scalar components of the second Piola-Kirchhoff stress in the basis $\boldsymbol{X}_{\alpha} \otimes \boldsymbol{X}_{\beta}$ with $\alpha=1,2$ and $\beta=1,2$. The Jacobian determinant is defined as $\sqrt{G}=\boldsymbol{X}_{, 1} \cdot\left(\boldsymbol{X}_{, 2} \times \boldsymbol{X}_{3,3}\right)$.

Recently, in [15], we introduced a method based on overlapping paired elements (applied to 4 node quadrilaterals) to represent crack propagation in thin shells that allows a relatively direct implementation of element-wise fracture in plates and shells. The method uses two distinct deformation maps, one for each part of the cracked shell. In pristine regions, the deformation maps coincide. A signed distance function $g(\boldsymbol{X})$ is adopted to identify the crack core $\Gamma_{c}$.

In the discretization by finite elements, the degrees of freedom are mid-side rotations and corner-node displacements. Local duplication of homologous degrees of freedom is used in cracked elements. The basis functions agree with those of the extended finite element method (e.g. [20, 21, 22]), but a different perspective is taken. Additionally, in the present context, 4 additional degrees of freedom per element are used corresponding to the cohesive stresses. 
Note that the directors in the deformed configuration follow independent deformation maps. Using the signed distance to the crack surface, $g(\boldsymbol{X})$ such that $g(\boldsymbol{X})<0$ for $\boldsymbol{X} \in \Omega^{-}, g(\boldsymbol{X})>0$ for $\boldsymbol{X} \in \Omega^{+}$and $\boldsymbol{X}=0$ for $\boldsymbol{X} \in \Gamma_{c 0}$ we have $\Gamma_{c 0}=\left\{\boldsymbol{X}_{c} \in \mathbb{R}^{3}: g\left(\boldsymbol{X}_{c}\right)=0\right\}$.

The shell element used in the discretization contains 4 corner nodes where the mid-surface positions are interpolated and 4 mid-sides where rotations are interpolated. Implementation details are given in reference [15]. For completeness, we provide the deformation map (in $\Omega$ ):

$$
\boldsymbol{x} \equiv \boldsymbol{\varphi}(\boldsymbol{X})= \begin{cases}\underbrace{\boldsymbol{r}^{+}(\boldsymbol{\theta})+\theta^{3} \boldsymbol{n}^{+}(\boldsymbol{\theta})}_{\boldsymbol{x}^{+}}, & g(\boldsymbol{X})>0 \\ \underbrace{\boldsymbol{r}^{-}(\boldsymbol{\theta})+\theta^{3} \boldsymbol{n}^{-}(\boldsymbol{\theta})}_{\boldsymbol{x}^{-}}, & g(\boldsymbol{X})<0\end{cases}
$$

where $\boldsymbol{r}$ is the mid-surface position and $\boldsymbol{n}$ the deformed director field.

The Cauchy Lemma and the cohesive use specific quadrature points of the extended finite element method (XFEM). The tip advance criterion is the internal force at the tip.

\section{NON-SMOOTH COHESIVE LAW AND THE MIXED METHOD}

\subsection{Introduction to cohesive traction-separation laws}

The concept of cohesive force by Barenblatt [23, 24] (a review is presented in reference [1]) implies the finiteness of the stress caused by canceling of the singularity in the Westergaard asymptotic field with the singularity created by a cohesive tip. A depiction of this concept is provided in figure 2 where the mobile equilibrium crack (see Barenblatt [1] and Goodier [25]) is represented. The position of points $A$ and $B$ is obtained as part of the solution of the constrained boundary-value problem (BVP) stated in (1). The fracture process zone (FPZ) is associated with a fracture stress $f(x, d)$ where $x$ is the position along the crack and $d$ is the opening at a given position. Point $A$, which is the infimum in $x$ of the support of the cohesive force function $f(x, d)$, is a function of the opening displacement. Point $B$ is distinct: its motion is a consequence of equilibrium (hence the name equilibrium crack) by the imposition of a null stress intensity factor. Other conditions are equivalent to this one: the maximum stress ahead of the tip or the cusp-type shape of the opening profile of a cohesive crack. A zero force to close the tip is also an equivalent in the discrete setting.

We direct the reader to the textbook by Bažant and Planas [3] for a description and the paper by Moës and Belytschko [26] for an application of this concept with the extended finite element method. It is worth noting that cohesive laws are subject to the condition that contact complementarity should be obtained as a limit case (null fracture energy and maximum stress). Smoothing of the contact linear complementarity problem (LCP) has been performed by Eterovic and Bathe [27].

The numerical treatment of cohesive laws appears to be less studied than the applications. Originally, regularization was employed in the loading/unloading region of the curves and often intrinsic (and in certain cases holonomic, [26]) laws were employed in numerical applications. Smooth loading laws were used by $\mathrm{Xu}$ and Needleman [28] but do not constitute a solution for the implementation in a displacement-based context.

After the papers by Falk et al. [29] and Alfano and Crisfield [6] it became clearer that 


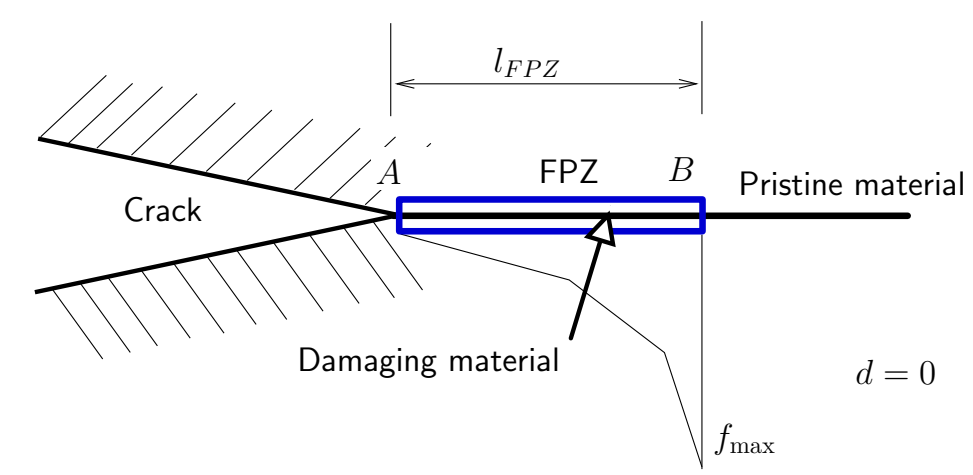

Points $A$ and $B$ move and define the configuration

Figure 2. The mobile equilibrium crack (see $[1,25]$ ) and the fracture process zone (FPZ): points $A$ and $B$ move according to the released energy and are here denoted configurational points.

results of initially rigid laws are different from those obtained with regularization, namely in the multi-fracture and bifurcation contexts (see also [8]). Furthermore, very rigid laws induce greater condition numbers of the Jacobian matrix in an implicit solution framework. The cohesive problem with unloading is non-holonomic, which means that the conditions can only be written with rate kinematical quantities (this also occurs with elasto-plasticity or when two rigid objects contact in rolling [30]).

The identification of this problem as a complementary problem appears to have been first done by Bolzon et al. [11] (a more complete analysis was performed by Tin-Loi and Tseng [31]). However, the implementations are not amenable to be incorporated in classical finite element codes. The authors in [31] have dealt exclusively with holonomic mode I, despite having a correct representation of the initial infinite slope. The authors in [11] used a rigid-plastic law, which resulted in a discrete variational inequality analogous to the one of elasto-plasticity and hence the same algorithms could be employed. More recently, Tin-Loi and Que [32] performed a comparison (limited to the holonomic case) between several methods to solve a LCP and concluded that smoothing methods are appropriate from both the accuracy and efficiency viewpoints. One striking fact in these tests is that no special treatment of the initial infinite slope was discussed, which is the most troublesome aspect in modeling cohesive laws. We try to avoid the simplifications in these works and will see that the complete non-holonomic treatment makes use of a separate inspection of two degenerate cases.

We review the typical initially rigid cohesive law as depicted in figure 3 . The area enclosed between the initial loading curve and the $d$ axis equals the critical fracture energy $G_{c}$, as deduced by use of the $\mathrm{J}$ integral (e.g. see page 167 in [33]). We note that two complementarity conditions exist for non-degenerate cases. For degenerate cases, only one complementarity condition exists. The contact/unloading/reloading curve is valid for $f<f_{\max }$ and $d \leq \widetilde{d}$ and the loading/stress free curve is valid for $d>\widetilde{d}$ or $f \geq f_{\max }$.

The existence of the two degenerate cases and a set-valued force inhibits the use of standard displacement-based methods. Note that the second degenerate case corresponds to the contact LCP and occurs with $\widetilde{d}=d_{c}$. It is also noteworthy that if $G_{c}=0$, it follows that $d_{c}=0$ and

Int. J. Numer. Meth. Engng 2004; 00:1-6 
no characteristic length exists. However, a characteristic force exists $\left(f_{\max }\right)$. In such case, the drop $f_{\max } \rightarrow 0$ occurs instantaneously, with debonding of the cohesive surface. The switch condition between these two conditions is an added complication that was not, to the authors' knowledge, been treated before.

A method to solve this problem consists in smoothing both the complementarity conditions and the switch condition and perform the iteration in the force-displacement space. This increases the total number of degrees of freedom but results in a well conditioned algebraic (non-symmetric) problem. We make use of a trust region algorithm (see also [34]) to solve the constrained equilibrium problem.

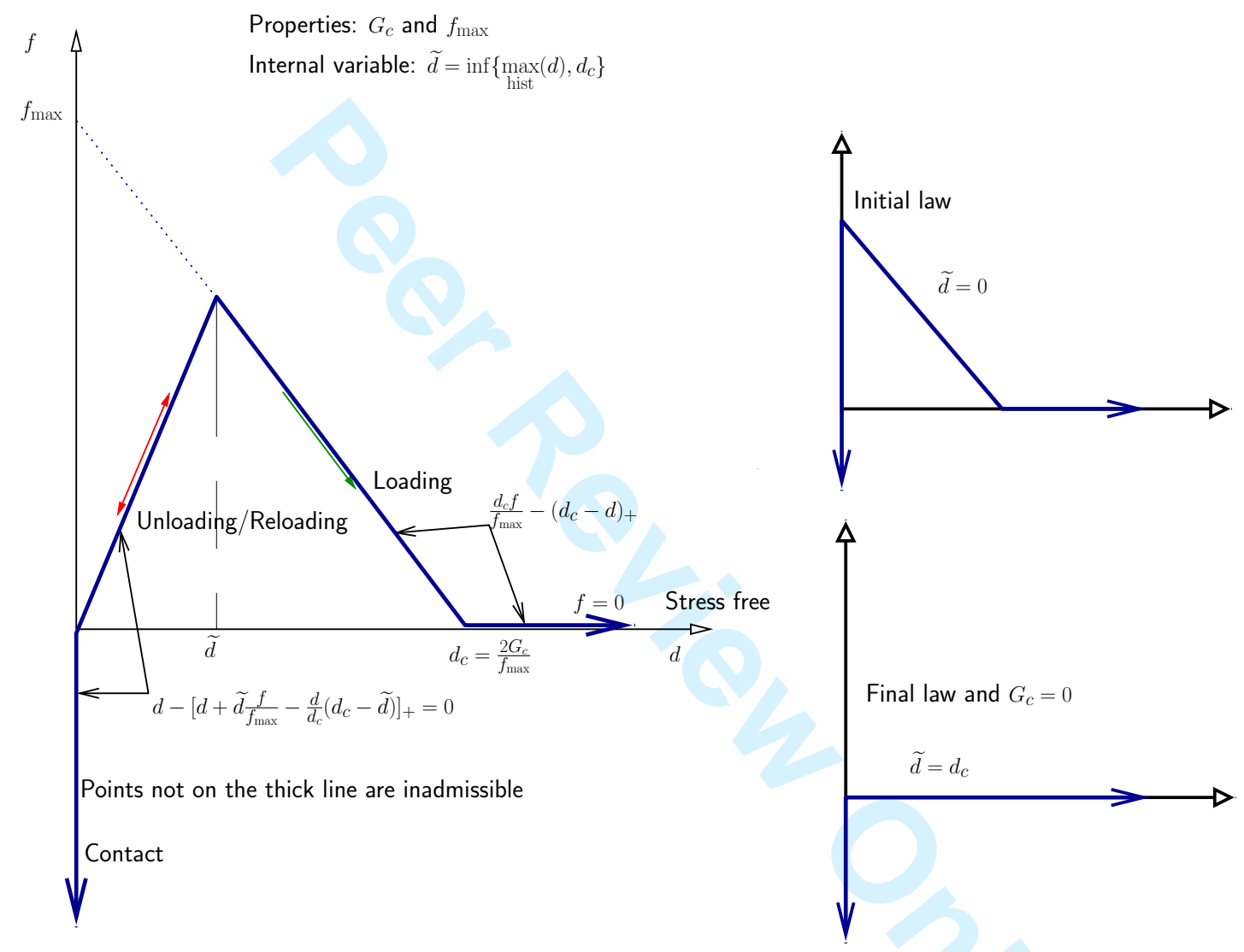

Figure 3. The prototype model for the cohesive law. Unloading occurs to the origin. At a given time step, the internal variable $\widetilde{d}$ is fixed. Two degenerate cases are shown: the initial law and the contact problem.

\subsection{Effect of smoothed functions}

Replacement of the "step" and "plus" functions by smooth approximations allow the use of Newton-based root-finding algorithms. We view smoothing as distinct of regularization, as 
the latter results in additional stiffness in the algebraic system. The purpose of smoothing is the replacement of complementarity conditions and other non-smooth conditions (such as branches) by more tractable constraints in the force (or stress)-displacement space.

For the linear case a perturbed method was proposed by Chen and Harker [35] where the orthogonality condition was relaxed. With smoothing methods this condition is also relaxed. For the non-linear case, it was found by Chen and Mangasarian [17] that, under certain conditions, the smooth problem is solvable and has as limit the solution of the NCP.

We make use of part of the formulation established in [17] where a method is proposed to solve nonlinear (and also mixed) complementarity problems. Instead of the line-search used in their work, we here use a trust region method because the crack opening modifies the radius of convergence of the Newton method, as will be shown.

Chen and Mangasarian [17] provide a catalog of smooth replacement functions. We direct the reader to the proofs by Chen and Harker [18] and Chen and Mangasarian [17]. In particular, as a relation is established with interior point methods, proofs concerning the iteration trajectory make use of similar conclusions.

A smooth modification of the step function is given by $(x \in \mathbb{R})$ :

$$
s(x, \alpha)=\frac{1}{1+\mathrm{e}^{-\alpha x}}
$$

where the parameter $\alpha$ can be calculated for a given tolerance. We prescribe tol $=x_{s=1-\epsilon}-x_{s=\epsilon}$ and obtain:

$$
\alpha=\frac{2}{\operatorname{tol}} \ln \left(\frac{1-\epsilon}{\epsilon}\right)
$$

This smooth function can be derived using probability density functions. Further properties are provided in references $[17,18]$. The corresponding smoothed "plus" function is given by integration of $s$ :

$$
p(x, \alpha)=x+\frac{1}{\alpha} \ln \left(1+\mathrm{e}^{-\alpha x}\right)
$$

with $p[x, \alpha(\mathrm{tol})] \leq x_{+}+\frac{\log (2)}{\alpha(t o l)}$ where $x_{+}=\max (0, x)$. Note that the form of the functions (5) and (7) is adequate for $x>0$ but should be modified for $x<0$ to avoid floating point overflow. The selection of these functions is not irrelevant for the solution. Other methods can be generated using distinct functions. For example, an interior point method is generated by selecting an interior point smoothing function:

$$
p_{\text {ip }}(x, \alpha)=\frac{x+\sqrt{x^{2}+4 / \alpha}}{2}
$$

As alluded before, caution should be taken in the use of smoothed functions to avoid "spikes". For example, for a branch function such as $y=x / c_{1}: x<c_{1}, y=(1-x) /\left(1-c_{1}\right): x \geq c_{1}$ with $x \in[0,1]$ and $c_{1} \in[0,1]$, the maximum of $y$ in this interval is 1 , however, the corresponding smoothed function $y_{s}=p\left(c_{1}-x\right) x / c_{1}+p\left(x-c_{1}\right)(1-x) /\left(1-c_{1}\right)$ is unbounded as $c_{1} \rightarrow 0$.

The smooth replacements must also ensure that the solution of the original problem is the same as the solution of the replacement problem as tol $\rightarrow 0$. It is clear that $x_{+}=$ $\lim _{\alpha \rightarrow+\infty} p[x, \alpha]$. However this should occur with the solution of the NCP problem, if exists. 
Chen and Mangasarian [17] and, more recently Chen and Harker [18] have shown that it is the case under the conditions given below. The statement of the NCP is:

Find $\boldsymbol{x}$ in $\mathbb{R}^{n}$ such that $x_{i} \geq 0, G\left(x_{i}\right) \geq 0$ and $G\left(x_{i}\right) x_{i}=0$. This is equivalent to $x_{i}=\left[x_{i}-G\left(x_{i}\right)\right]_{+}$

where $\boldsymbol{G}(\boldsymbol{x})$ is a differentiable function from $\mathbb{R}^{n}$ to $\mathbb{R}^{n}$. Note that, due to non-negativity of the components, it follows that $G\left(x_{i}\right) x_{i}=0 \Leftrightarrow \boldsymbol{x} \cdot \boldsymbol{G}(\boldsymbol{x})=0$.

A NCP is said to be strongly monotone if

$$
\exists k \in \mathbb{R}^{+}: \forall \boldsymbol{x}, \boldsymbol{y} \in \mathbb{R}^{n} \quad[\boldsymbol{G}(\boldsymbol{x})-\boldsymbol{G}(\boldsymbol{y})] \cdot(\boldsymbol{x}-\boldsymbol{y}) \geq k\|\boldsymbol{x}-\boldsymbol{y}\|^{2}
$$

It is known that a strongly monotone NCP has a unique solution (see [18]).

For a Lipschitz continuous $G(\boldsymbol{x})$ (i.e. $\exists K \in \mathbb{R}: \quad\|G(\boldsymbol{x})-G(\boldsymbol{y})\| \leq K\|\boldsymbol{x}-\boldsymbol{y}\|, \quad \forall \boldsymbol{x}, \boldsymbol{y} \in \mathbb{R}^{n}$ ) and strongly monotone NCP, let $\boldsymbol{x}(\alpha)$ be a solution of $x_{i}-p\left[x_{i}-G\left(x_{i}\right), \alpha\right]=0, i=1, \ldots, n$. Then, for the solution $\overline{\boldsymbol{x}}$ of the NCP it follows that:

$$
\exists C_{p} \in \mathbb{R}_{+}: \quad\|\boldsymbol{x}(\alpha)-\overline{\boldsymbol{x}}\|_{p} \leq C_{p} \gamma_{p} \log (2) / \alpha, \quad p=1,2, \infty
$$

with $\gamma_{1}=n, \gamma_{2}=\sqrt{n}$ and $\gamma_{\infty}=1$. The existence of a solution for the smoothed problem $(\boldsymbol{x}(\alpha))$ is still subject to proof under the previous conditions. For a solvable NCP, the following theorem (the proof is given by Chen and Mangasarian [17]) follows:

Theorem 1. Let the NCP be solvable. If $\delta_{1} \geq \log 2$ and $\alpha>0$, the NCP conditions are approximately satisfied as

$$
\begin{array}{r}
(-x)_{+} \leq \frac{\delta_{1}}{\alpha} \mathbf{1}, \quad(-\boldsymbol{G}(\boldsymbol{x}))_{+} \\
\text {with } \quad C\left(\delta_{1}\right)=\max \left\{2,\left(e^{\delta_{1}}-1\right) \log ^{2}\left(e^{\delta_{1}}-1\right)\right\}
\end{array}
$$

For the present applications, it remains to show that our NCP is strongly monotone. Even for Hookean elasticity, this condition cannot presently be ensured. For a LCP, Tin-Loi and Tseng [31] also mention this problem, but the application of the solution method does not appear to be affected.

\subsection{Treatment of a prototype cohesive law}

The set-valued cohesive law shown in figure 3 can be given by the following non-smooth equation:

$$
e q(d, f)=0
$$

A corresponding smoothed version for the non-degenerate case is introduced making use of the smoothing parameter $\alpha(t o l)$ in (6):

$$
e q_{s}[d, f, \alpha]=e q_{1}[d, f, \alpha] a b+e q_{2}[d, f, \alpha](1-a b)
$$


with

$$
\begin{aligned}
& a=s(\widetilde{d}-d, \alpha) \\
& b=s\left(d_{c}-d_{c} \frac{f}{f_{\max }}, \alpha\right)
\end{aligned}
$$

and

$$
\begin{array}{r}
e q_{1}[d, f, \alpha]=d-p\left[d+\widetilde{d} \frac{f}{f_{\max }}-\frac{d}{d_{c}}\left(d_{c}-\widetilde{d}\right), \alpha\right] \\
e q_{2}[d, f, \alpha]=p\left[d_{c}-d, \alpha\right]-\frac{d_{c} f}{f_{\max }}
\end{array}
$$

which, for $G_{c}=0.5$, and $f_{\max }=1$ is represented in figure 4 for several values of $\widetilde{d}$ and $t o l$.

The gradient of $e q_{s}(d, f)$ can be calculated using the chain rule. This is performed with a computer algebra software. However, it is interesting to represent it in the space $d-f$ for our specific material properties (see figure 5 ) with $t o l=0.5$ and $\widetilde{d}=0.2$. Note that the derivatives are also smooth, and a therefore a (non-symmetric) smooth solver can be used to solve the algebraic nonlinear system:

$$
\boldsymbol{F}=\left\{\begin{array}{c}
r_{\text {equ. }}(\boldsymbol{u}, \boldsymbol{f}) \\
e \boldsymbol{q}_{s}[\boldsymbol{d}(\boldsymbol{u}), \boldsymbol{f}]
\end{array}\right\}=\mathbf{0}
$$

for $\boldsymbol{u}$ and $\boldsymbol{f}$. In the system (14), $\boldsymbol{r}_{\text {equ. }}$. represents the vector of discrete equilibrium equations ( $n$ components), $\boldsymbol{e} \boldsymbol{q}_{s}$ represents the vector of smoothed conditions ( $m$ components). The nonlinear system with $m+n$ unknowns is solved by the trust region method shown in table II, see also [34]. The degrees of freedom corresponding to $\boldsymbol{f}$ and $\boldsymbol{d}$ are scaled (with the conditions $f \neq f_{\max }$ and $d \neq d_{c}$ ) so that the cohesive law is the one depicted here.

The nonlinear solver makes use of the Jacobian of (14), which is generally non-symmetric:

$$
\boldsymbol{K}=\left[\begin{array}{cc}
\frac{\partial \boldsymbol{r}}{\partial u} & \frac{\partial \boldsymbol{r}}{\partial f} \\
\frac{\partial \boldsymbol{e} \boldsymbol{q}_{s}}{\partial \boldsymbol{u}} & \frac{\partial \boldsymbol{e q}_{s}}{\partial \boldsymbol{f}}
\end{array}\right]
$$

The number of degrees of freedom and sparsity pattern of (15) change during the solution.

A characteristic which is different from previous implementations of the cohesive law in the context of XFEM, is that caution should be taken to avoid duplication of constraints. Note that after exhaustion of the cohesive law $\left(\widetilde{d}>d_{c}\right)$ the diagonal term $f-f$ of the Jacobian is equal to 1 , and therefore no elimination of the corresponding $f$ degree-of-freedom is required. The system (14) is solved by a monolithic approach.

A simple test of the use of the trust region method and fixed time steps is depicted in figure 6 .

Note that there are no signs of ill-conditioning, but the method entails the costs of a nonsymmetric Jacobian.

For $k=1000$, we test the sensitivity of the non linear solver with respect to the solution parameter tol and also the average number of time steps $n_{\text {step }}$. The monitored quantities are the average number of iterations $n_{\text {iter }}^{\text {av }}$ and the maximum number of iterations in a given step $n_{\mathrm{iter}}^{\max }$. The initial trust radius is 1 and a tolerance of $t o l_{t r}=1 \times 10^{-6}$ is employed for the trust region method. Table I shows the results. A near-insensitivity to the curvature is noted. 


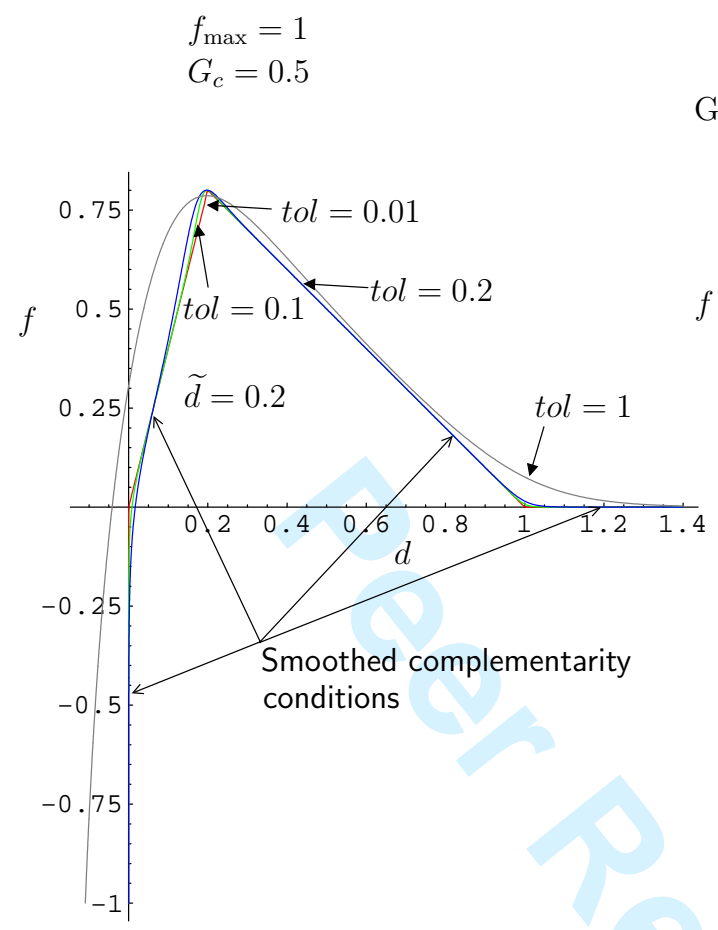

$$
\operatorname{Graf}\left\{e q_{s}[d, f ; \alpha(t o l)]=0\right\}
$$

Figure 4. Smoothed cohesive laws for various tol and $\widetilde{d}$.
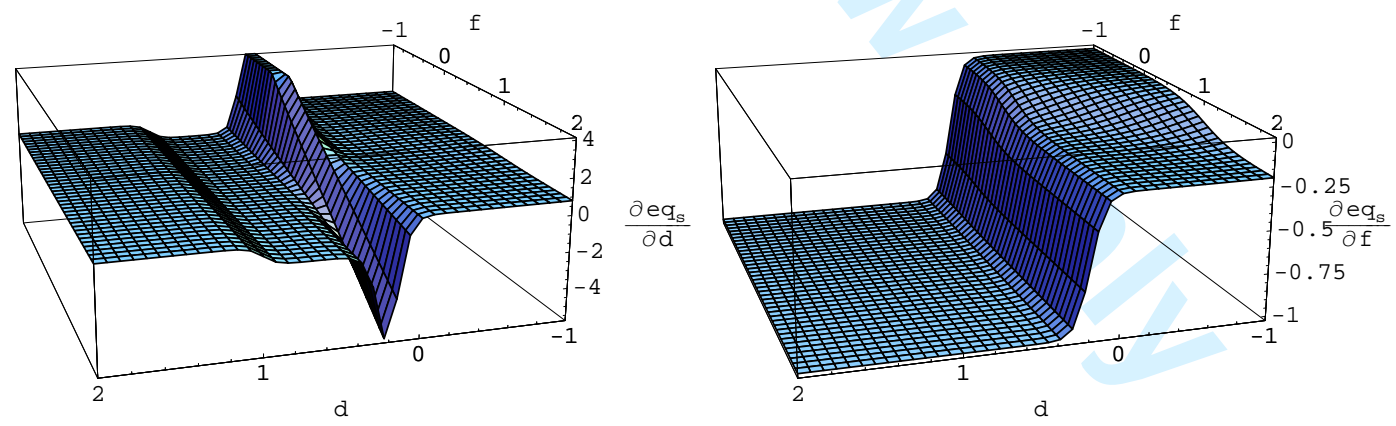

Figure 5. Smoothed cohesive laws: derivatives of the constraint $e q_{s}(d, f)=0$ with respect to $d$ and $f$ in the $d-f$ space. tol $=0.5$ and $\widetilde{d}=0.2$ 

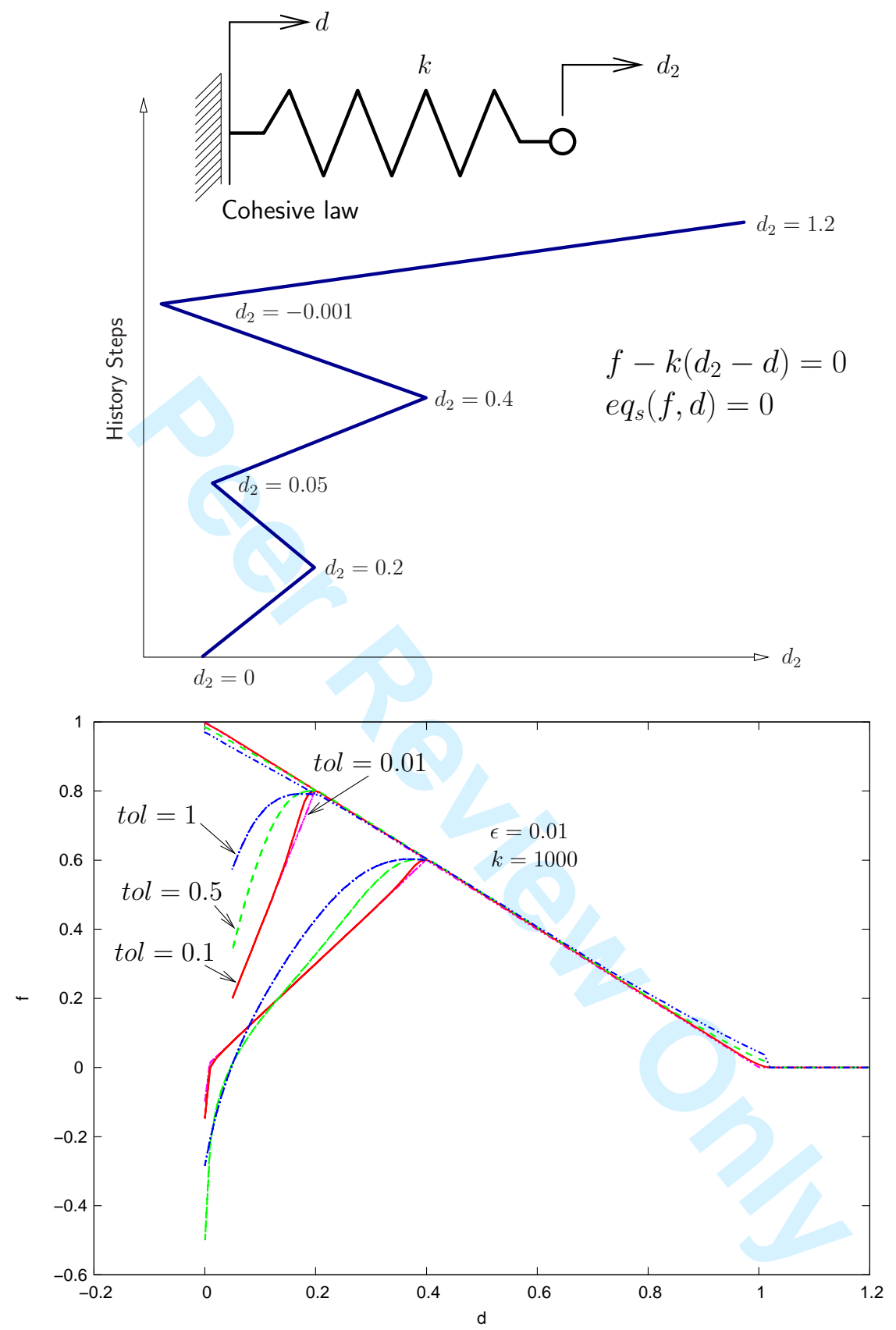

Figure 6. Effect of tol for a spring with an end cohesive law. 
Table I. Average number of iterations and maximum number of iterations in a given time step, as a function of $t o l$ and $n_{\text {step }}$. The relevant properties are $k=1000$ and $\epsilon=0.01$.

\begin{tabular}{cccc}
$n_{\text {step }}$ & $t o l$ & $n_{\text {iter }}^{\text {av }}$ & $n_{\text {iter }}^{\max }$ \\
\hline 300 Steps & 0.02 & 1.55 & 7 \\
& 0.05 & 1.72 & 9 \\
& 0.1 & 1.97 & 8 \\
& 0.5 & 2.10 & 6 \\
& 1 & 2.06 & 3 \\
& 2 & 2.03 & 3 \\
\hline 100 Steps & 0.02 & 1.63 & 7 \\
& 0.05 & 1.99 & 9 \\
& 0.1 & 2.22 & 9 \\
& 0.5 & 2.25 & 7 \\
& 1 & 2.28 & 4 \\
& 2 & 2.23 & 3 \\
\hline 50 Steps & 0.02 & 1.77 & 7 \\
& 0.05 & 2.22 & 9 \\
& 0.1 & 2.47 & 9 \\
& 0.5 & 2.55 & 7 \\
& 1 & 2.39 & 4 \\
& 2 & 2.39 & 4 \\
\hline
\end{tabular}

\section{CRACK ADVANCE CRITERION AND ORIENTATION}

For cohesive cracks, the crack advance problem is of more direct address than for non-cohesive cracks. From a strict reading of Barenblatt's work, the advance of a crack is supported by the existence of an initial positive slope (and zero stress for zero displacement) in the stress-displacement law. We already observed that this is not consistent with a continuumdiscontinuum transition, as it would cause an abrupt unloading of neighbor regions.

Our theory for crack advance is based on the similarity (in loading) of the crack tip cohesive stress distribution (Barenblatt's second hypothesis and Goodier's third postulate [25]). When a new extension of the crack surface is required, we assume that, at the instant of surface creation, the new part of the cohesive stress cancels the pre-existent normal stress distribution. This is equivalent to assume that no stress jump occurs. From this condition we obtain both the orientation of newly formed crack surfaces and the crack advance. The former is calculated from the zero jump condition and the latter is obtained from the internal force required to close the tip. We introduce the condition for advance:

$$
f_{\text {tip }} \cdot e_{\mathrm{I}}<0
$$

where $\boldsymbol{f}_{\text {tip }}$ is the internal force at the tip, resulting from the combined effects of opening forces and the cohesive law. In loading, a cusp-shaped tip closing is expected to correspond to a null (resulting) stress intensity factor, as discussed by Barenblatt [1]. For sufficiently long cohesive tails (figure shows a typical tail 16), the traditional finite element resolution can be adequate 
Table II. Trust region method. The degrees of freedom $u_{i}$ and $f_{j}$ are grouped in array $\boldsymbol{z}$.

\begin{tabular}{|c|c|}
\hline Step & Tasks \\
\hline & Initialization \\
\hline Data & $t_{\text {tol }}, R_{0}$ \\
\hline Iteration counter and initial radius & $n_{\text {iter }}=0, R=R_{0}$ \\
\hline & Function evaluation \\
\hline Store previous values for $n_{\text {iter }}>0$ & $\mathfrak{f}_{\text {old }}=\mathfrak{f}$ \\
\hline & $\mathfrak{m}_{\mathrm{old}}=\mathfrak{m}$ \\
\hline Evaluate the residual & $\boldsymbol{F}$ \\
\hline Objective & $\mathfrak{f}=0.5 \boldsymbol{F} \cdot \boldsymbol{F}$ \\
\hline Jacobian of $F$ & $\boldsymbol{J}=\frac{\partial \boldsymbol{F}}{\partial \boldsymbol{z}}$ \\
\hline Jacobian of $\mathfrak{f}$ & $\boldsymbol{g}=\boldsymbol{J}^{0 \boldsymbol{2}} \boldsymbol{F}$ \\
\hline Temporary value & $p=J g$ \\
\hline & Update of radius \\
\hline Calculate merit for $n_{\text {iter }}>0$ & $\boldsymbol{r}_{d}=\boldsymbol{J} \boldsymbol{d}, \mathfrak{m}=\mathfrak{f}+\boldsymbol{d} \cdot \boldsymbol{g}+0.5 \boldsymbol{r}_{d} \cdot \boldsymbol{r}_{d}$ \\
\hline Calculate evolution ratio $n_{\text {iter }}>1$ & $\rho=\frac{\mathfrak{f}_{o l d}-\mathfrak{f}}{\mathfrak{m}_{o l d}-\mathfrak{m}}$ \\
\hline Update the radius for $n_{\text {iter }}>1$ & $\rho<0 \rightarrow R=0.5 R$ \\
\hline & $0<\rho \leq 0.95 \rightarrow R=\left[0.5+0.5\left(\frac{\rho}{0.95}\right)^{2}\right] R$ \\
\hline & $\rho>0.95 \rightarrow R=\left[1.01+0.99 e^{-\left(\frac{\rho-1}{0.95-1}\right)^{2}}\right] R$ \\
\hline & Step determination \\
\hline Newton step & $\boldsymbol{d}^{N}=-\boldsymbol{J}^{-1} \boldsymbol{F}$ \\
\hline Verify Newton step & $\begin{array}{l}\left\|\boldsymbol{d}^{N}\right\| \leq t o l_{t r} \rightarrow \text { declare success and exit } \\
\left\|\boldsymbol{d}^{N}\right\| \leq R \rightarrow \boldsymbol{d}=\boldsymbol{d}^{N} \text { go to update of unknowns }\end{array}$ \\
\hline Unconstrained step (Cauchy) & $d^{u}=-\left(\frac{g \cdot g}{p \cdot p}\right) g$ \\
\hline Verify unconstrained step & $\left\|\boldsymbol{d}^{u}\right\| \geq R \rightarrow \boldsymbol{d}=-\frac{R}{\|g\|} \boldsymbol{g}$ go to update of unknowns \\
\hline Dogleg & $\begin{array}{l}\text { Solve for } h \in[0,1]: a_{1} h^{2}+a_{2} h+a_{3}=0 \\
\text { with } a_{1}=\left\|\boldsymbol{d}^{N}-\boldsymbol{d}^{u}\right\|^{2}, a_{2}=\boldsymbol{d}^{u} \cdot\left(\boldsymbol{d}^{N}-\boldsymbol{d}^{u}\right), a_{3}=\left\|\boldsymbol{d}^{u}\right\|^{2}-R^{2} \\
\boldsymbol{d}=\boldsymbol{d}^{u}+h\left(\boldsymbol{d}^{N}-\boldsymbol{d}^{u}\right)\end{array}$ \\
\hline & Update of unknowns \\
\hline Make temporary update & $z \leftarrow z+d$ \\
\hline$n_{\text {iter }}$ & go to function evaluation \\
\hline
\end{tabular}

to represent this cusp. We verify this in a plate bending numerical examples.

To obtain the orientation of a pre-existent cohesive crack, we make use of the fact that no jump in stresses should occur when the surface is formed. With a cohesive law, if we have pure mode $I$, this is equivalent to have zero tangential displacement, as a non-zero value would mean that a tangential stress would be released upon crack advance. The same terms can be applied for mode $I I$ and $I I I$. This criterion is of an extraordinary simplicity; an illustration is given in figure 7 . This can be directly obtained from the crack tip opening displacement components decomposed along each of the directions represented in the figure. Details about how this is performed are given by Sutton et al. [36] for a somehow different situation (the 
Erdogan-Sih criterion).

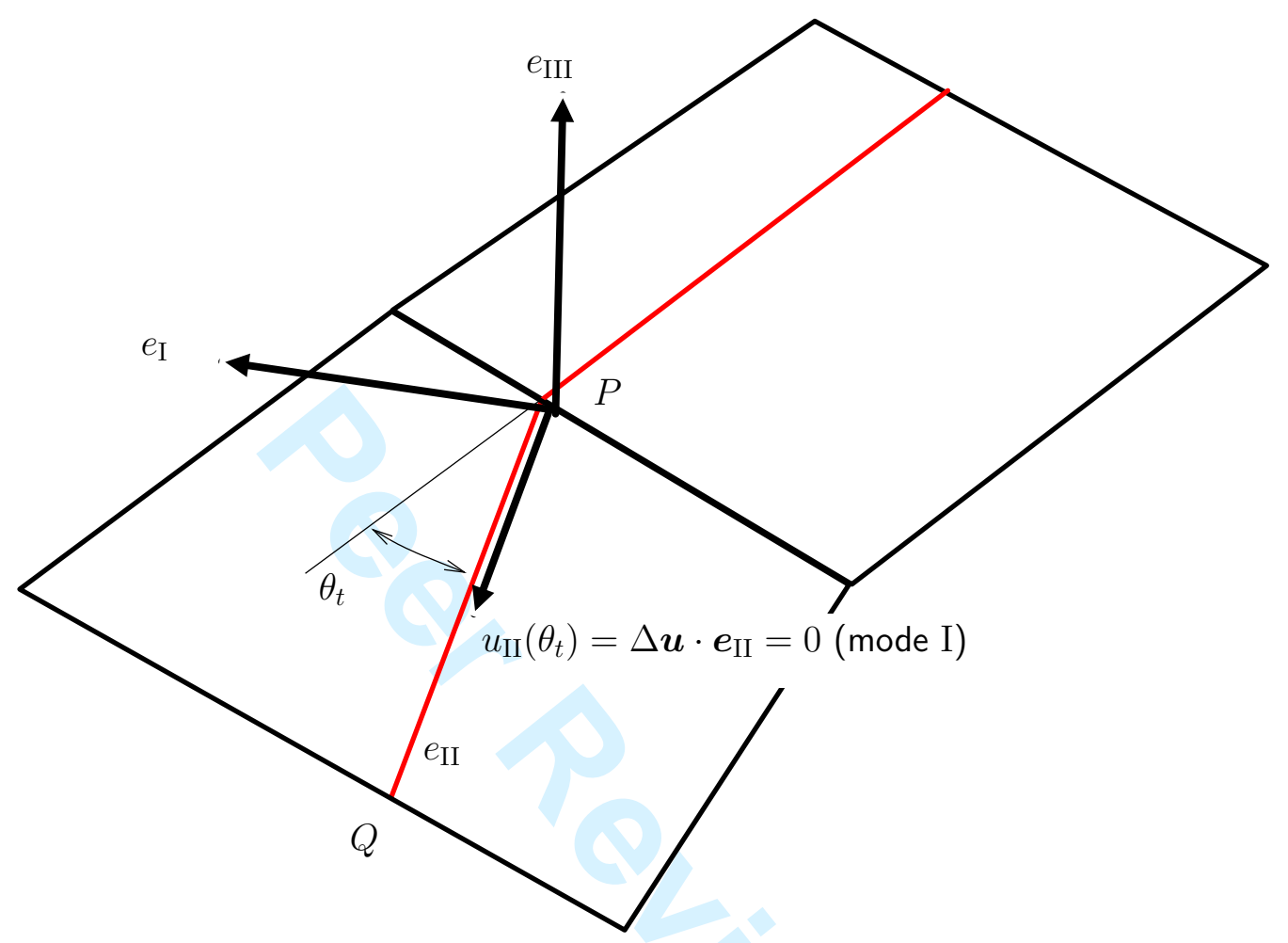

Figure 7. An example for mode I crack orientation based on zero stress jump.

For the elasto-plastic case (not discussed here), the COD concept is still applicable, whereas the application of configurational integrals is subject to certain conditions (such as inhibition of unloading).

\section{NUMERICAL EXAMPLES}

Results from verification tests are presented. The examples serve to inspect the main ingredients of the method, and also the implementation. In post-processing, elements are divided to create realistic crack evolution pictures.

Elasticity is considered (using the Saint-Venant model) and the piece-wise linear cohesive model with $t o l=0.01 d_{c}$. For the trust region method we use a tolerance of $t o l_{t r}=1 \times 10^{-6}$. Small enough load (and displacement) steps are used to avoid spurious crack holding. The advance can occur for any number of elements ahead of the tip. 


\subsection{In plane bending of a beam}

A rectangular beam subject to in-plane loading is tested for several values of the fracture energy. The overall response and the effect of mesh refinement are inspected. This test is essentially a plane stress situation in mode $I$ as rotations and out-of-plane motion are prescribed (the independent opening of top and bottom surfaces is performed in the subsequent example). The relevant properties are shown in figure 8. A number of features is intentionally left out from this test, so that conclusions concerning the cohesive law can be obtained without added complications.

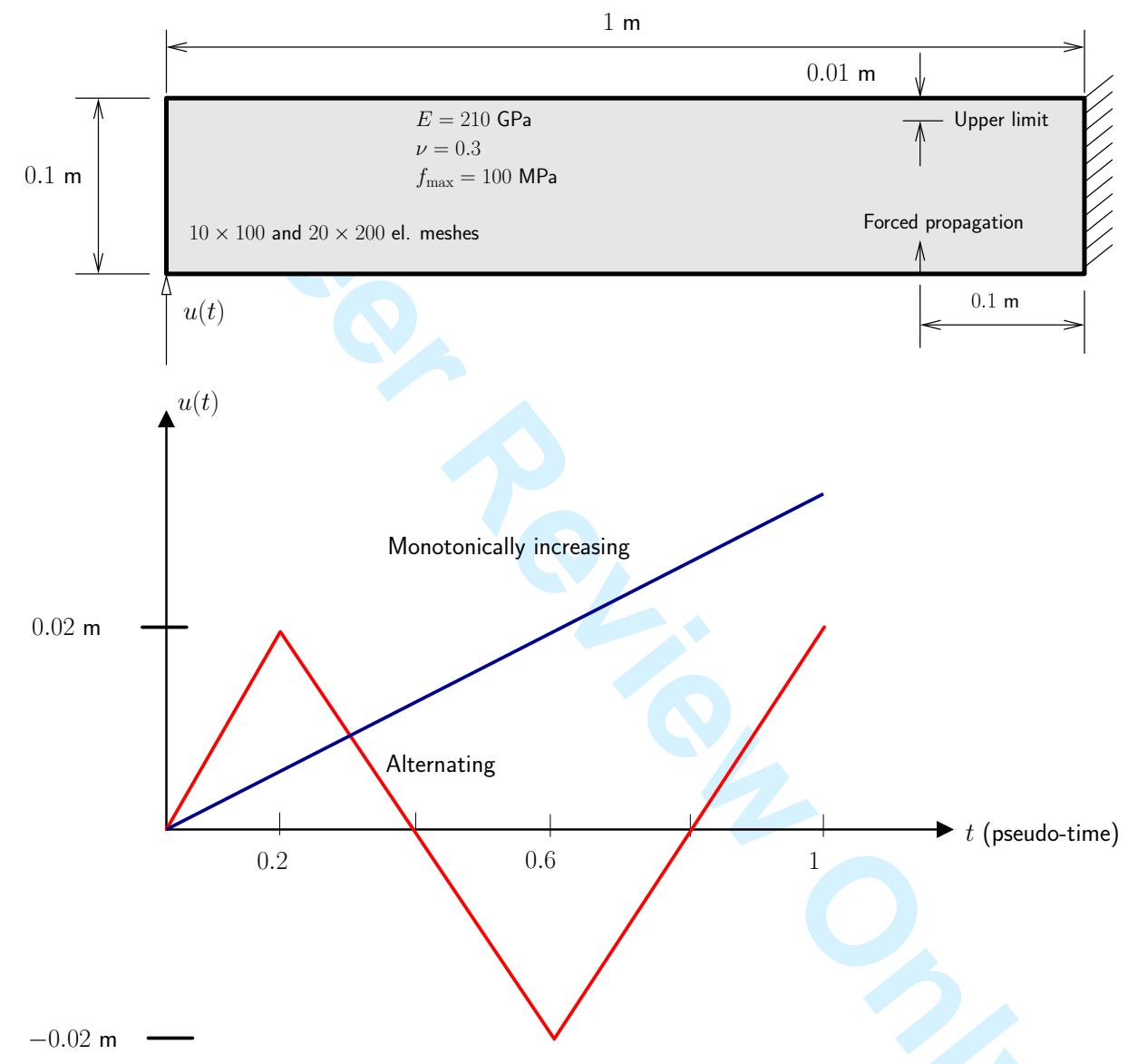

Figure 8. Beam bending under cyclic loading. Relevant properties, boundary conditions and geometrical data.

With a monotonic imposed displacement $u(t)=u_{0} t$, the load-deflection results are shown in figure 10 for 3 values of the fracture energy $G_{c} \in\{0,5000,20000\} \mathrm{N} / \mathrm{m}$. For verification purposes, in this example the Rankine criterion is adopted. The evolution of the radius of the trust region method is depicted in figure 11 for the cases $G_{c}=5000 \mathrm{~N} / \mathrm{m}\left(d_{c}=0.1 \mathrm{~mm}\right)$ and

Int. J. Numer. Meth. Engng 2004; 00:1-6 
$G_{c}=20000 \mathrm{~N} / \mathrm{m}\left(d_{c}=0.4 \mathrm{~mm}\right)$. The radius value is reset every time step. Clearly, we can see why classical Newton implementations (even with line search) are inefficient for NCP and LCP (such as elasto-plasticity). The combination of Cauchy step with the Newton step allows a robust solution without step cuts. Note that small steps are used to ensure accuracy in the integration. Any step size can be used, and the method never failed to converge during our test session, but some preliminary convergence study is advised.

Another revealing test can be performed by starting the enrichment before the stress reaches $f_{\max }$. The points in the $d-f$ diagram should be in the vertical loading region $d=0, \quad f \leq f_{\max }$ and therefore the results should not be affected by the early enrichment. The same cannot be said about delayed enrichment. For the finer mesh and $G_{c}=5000 \mathrm{~N} / \mathrm{m}$ this fact is illustrated in figure 9. We can see that premature enrichment is not critical (for proportional loading), confirming the correct implementation of the degenerate situation shown in figure 3 . The two classical methods to introduce a cohesive law (either holonomic or regularized) are obviously unable to provide this type of immunity to premature enrichment.

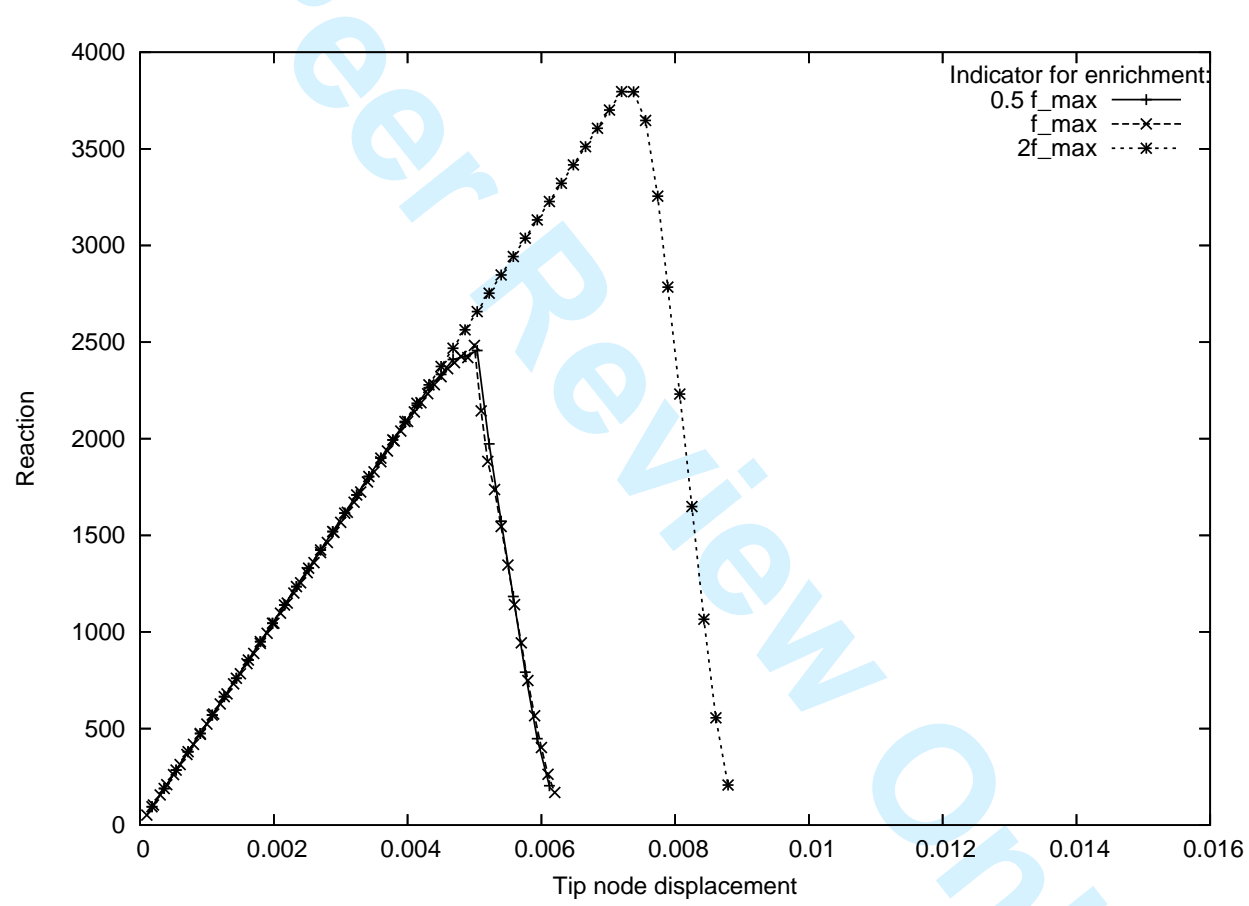

Figure 9. Effect of premature and delayed enrichment time in the load-deflection results $\left(G_{c}=5000\right.$ $\mathrm{N} / \mathrm{m}$ )

For higher values of the critical energy release rate, a decrease of the stress at the tip is observable when premature enrichment is forced.

For an imposed end-displacement of the piecewise linear type, the load-deflection results are shown in figure 12. Also shown are the (averaged) stress forces $f$ at the elements containing a crack for the case $G_{c}=20000 \mathrm{~N} / \mathrm{m}$ with a $10 \times$ magnification. We can be observe the 


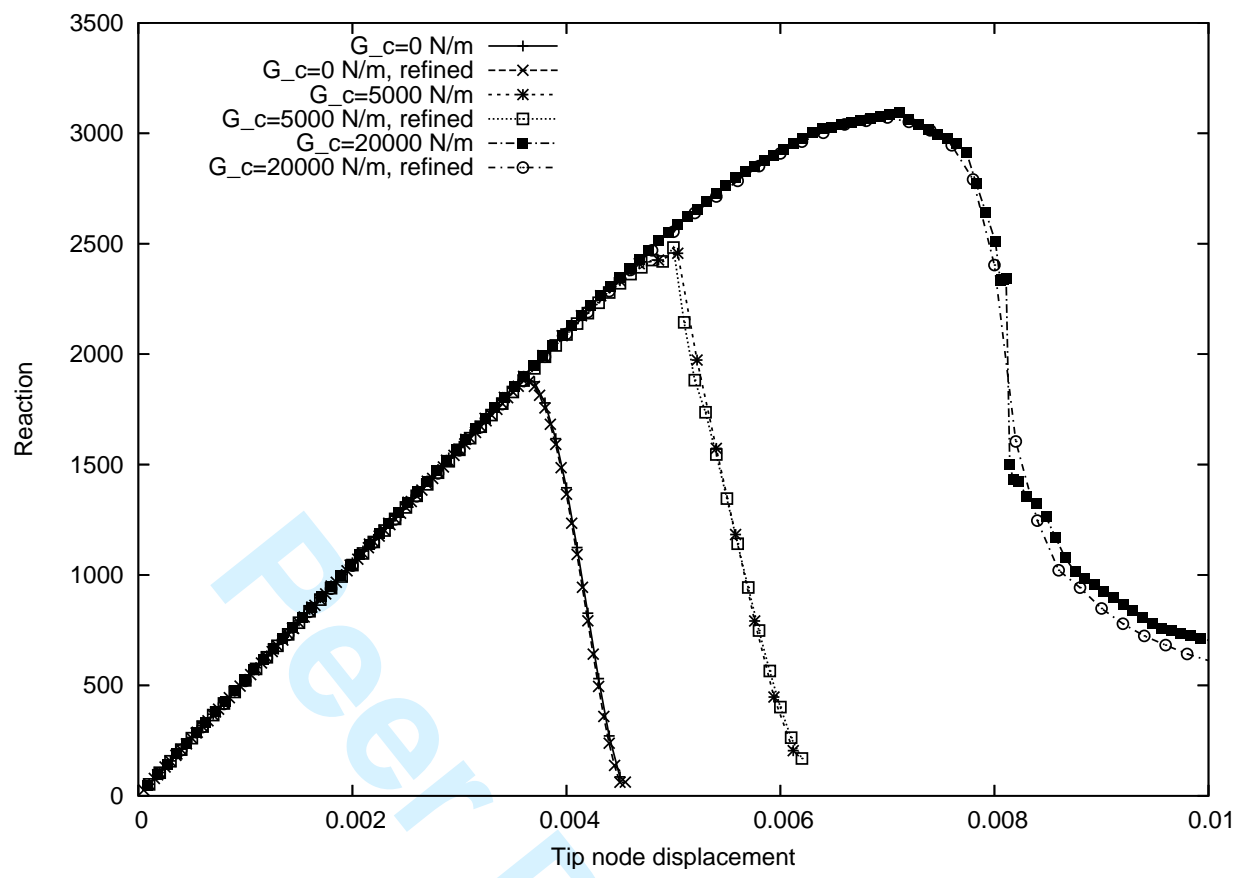

Figure 10. Beam under in-plane bending with monotonic end loading. Load-deflection results. The time steps are shown as point markings.

damage effect resulting from the cohesive law: the compliance is substantially increased after application of one load-reversal cycle. Another result to retain is that a very sharp curve is obtained in the reversals, which corroborates our indication that the problem is being correctly solved. No artificial detachments or spurious responses occurred. During the compression stage, it was noted that the cohesive force was zero in some points and hence some detachment should occur.

\subsection{Verification of the crack path and the cohesive stress}

An inspection of the $2 D$ crack path obtained with the proposed method is done using two known examples from the literature. The first example is a plain concrete fracture test (Gálvez et al. [37]) of a notched plate asymmetrically loaded by a vertical point load.

The problem is summarized in figure 13. The cracked beam and the tip detail are shown in figure 14. A comparison between the Rankine criterion (without averaging) and our model is presented in figure 15, along with the envelope of experimental results reproduced from [37]. In addition to the manifest good behavior in this linear-elastic problem, our criterion is not conditioned by the underlying constitutive model. In the implementation, the criterion is applied directly in the first intersection of the crack core with an element edge. The distribution of the cohesive force $f$ is given for a sequence of time steps in figure 16 .

The irregularity noted with the Rankine-calculated path is caused by the incompatible stress

Int. J. Numer. Meth. Engng 2004; 00:1-6 


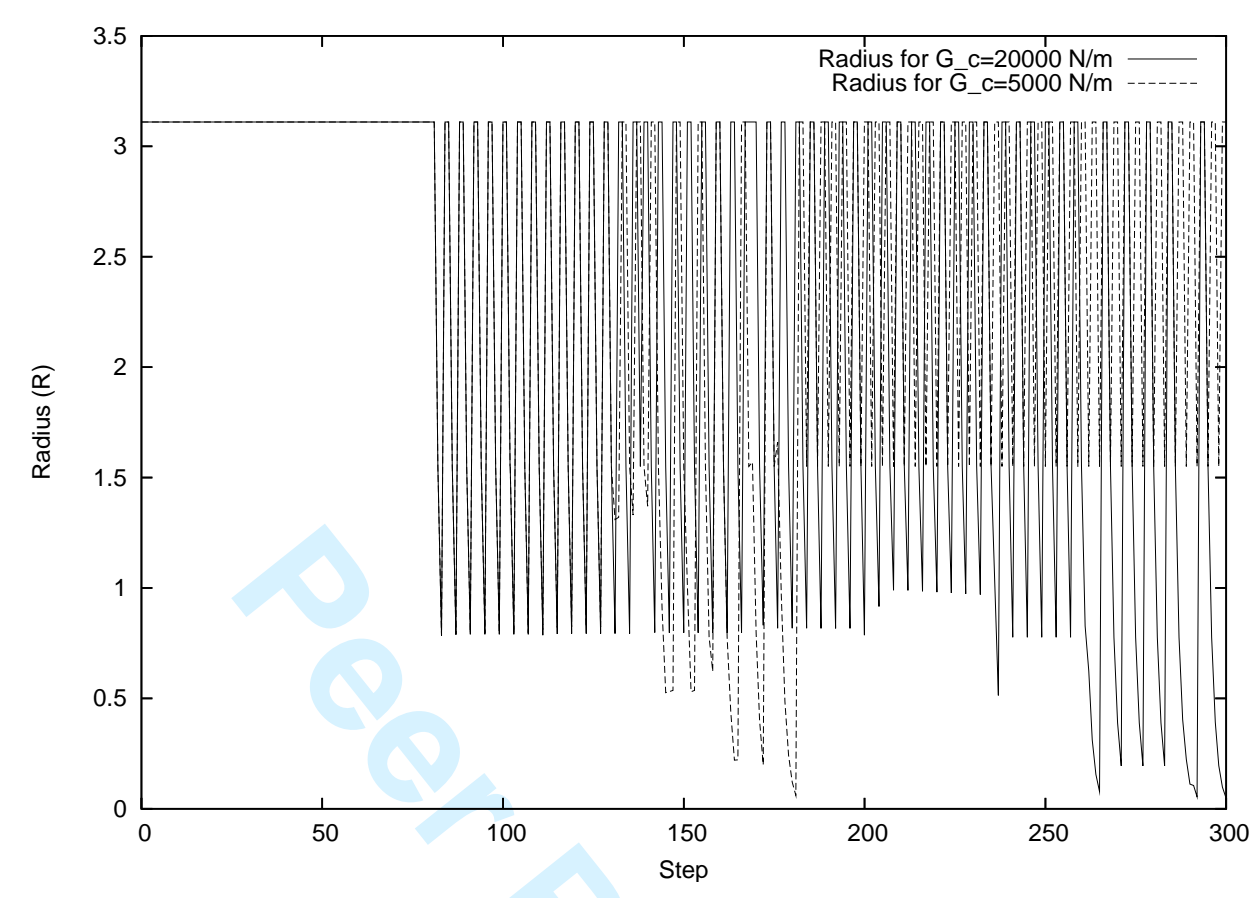

Figure 11. Beam under in-plane bending with monotonic end loading. Variation of the trust radius $(R)$ for all iterations and time steps.

field. An inversion of the crack path trajectory occurs and the analysis stops prematurely. This result of the Rankine criterion is not unforeseen.

The load-deflection curve is given in figure 17, along with the experimental envelope and the results from Areias and Belytschko [38] who used a $3 D$ model with regularized unloading. Note that averaging was used before by these authors to avoid the inversion of the crack path. A difficulty with averaging (this also applies to the domain integral) is the definition of domain size.

As the cohesive stress is an independent field, there is a complete decoupling with the continuum. In terms of root-finding convergence, the resulting properties are exceptionally good, as can be observed in figure 18. Usually one iteration is performed in each time step. The trust radius only reduces when closing/opening iterations occur. For comparison, a regularized cohesive law and the holonomic case are also shown. It is clear that, if we exclude unloading, then the problem is almost linear and the convergence behavior is very good before the discontinuous transition. When this is reached, the method fails to converge. In addition, although use can be made of the original Barenblatt condition in closed form (see the seminal work of Moës and Belytschko [26]), the model is incomplete. The regularized model also fails to converge prematurely, as shown in that figure.

The second example is a double-edge notched beam subject to two opposing opening forces and a third constant force in the right edge. This third force induces a smooth crack turning 


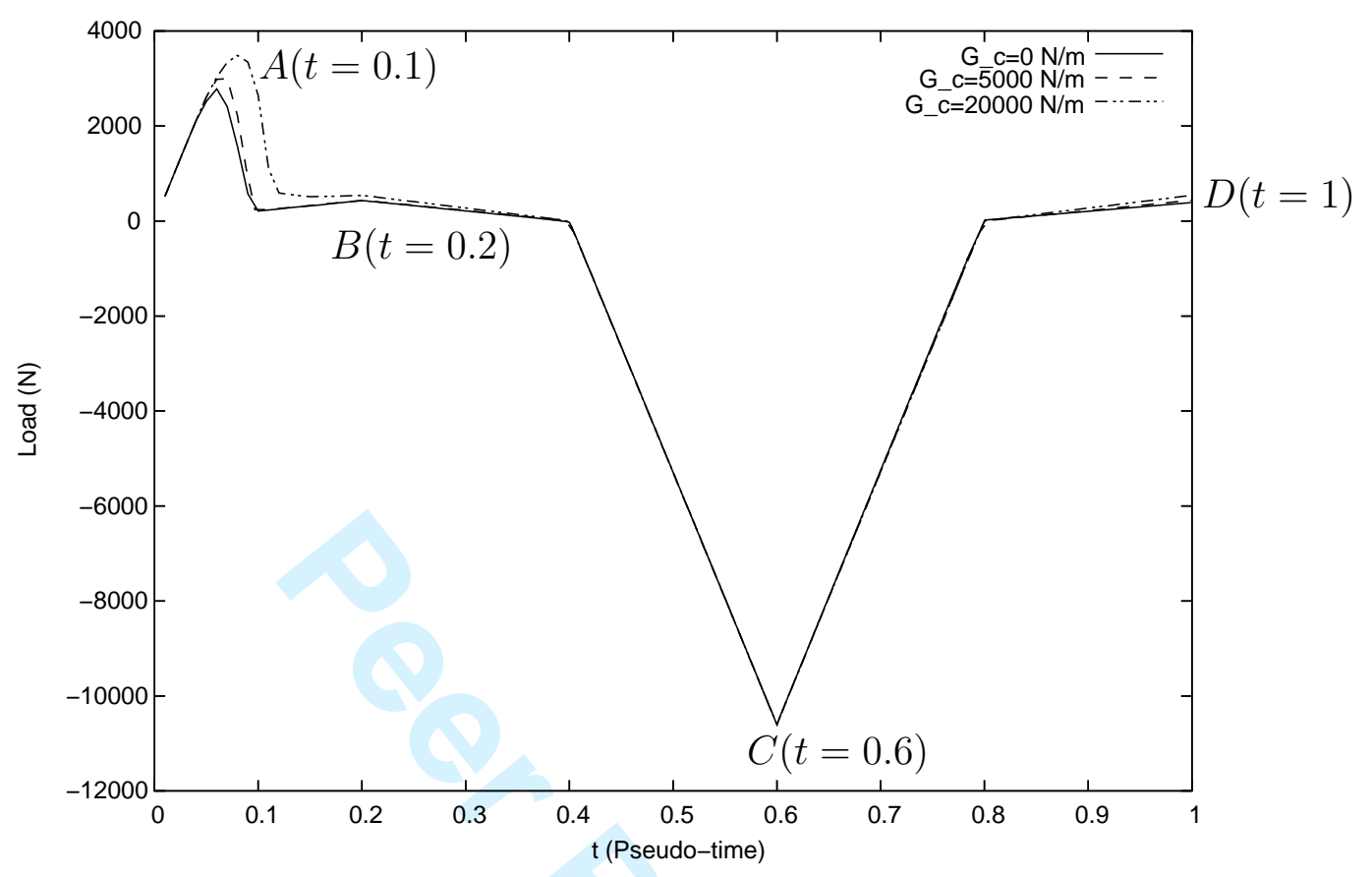

$A$
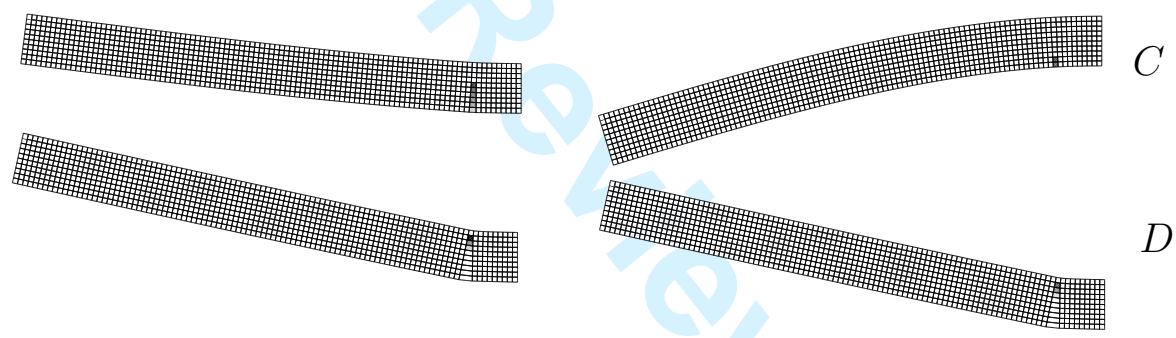

Figure 12. Beam under in-plane bending with oscillatory end loading. Load-deflection results and deformed meshes (magnified 10×). Also represented are the cohesive forces, with a maximum of 100 $\mathrm{MPa}$ for the tension cases and a minimum of $-534 \mathrm{MPa}$ for the compression case.

away from the notch orientation.

This example was initially proposed by Gálvez et al. [39] as a test for the accuracy of numerical implementations of crack growth. In that paper, the Erdogan-Sih criterion (see [33]) was used with success. As shown by Shen and Shen [40], this criterion coincides with the minimization of the potential energy with respect to the kink angle, for elastic isotropic materials.

However, it requires the values of the stress intensity factors (they can be calculated with a variety of techniques, such as the J-integral or extracted from the COD components) and also specialized tip elements. The present method, besides being applicable to general nonlinear situations, can be used with standard element formulations.

Int. J. Numer. Meth. Engng 2004; 00:1-6 


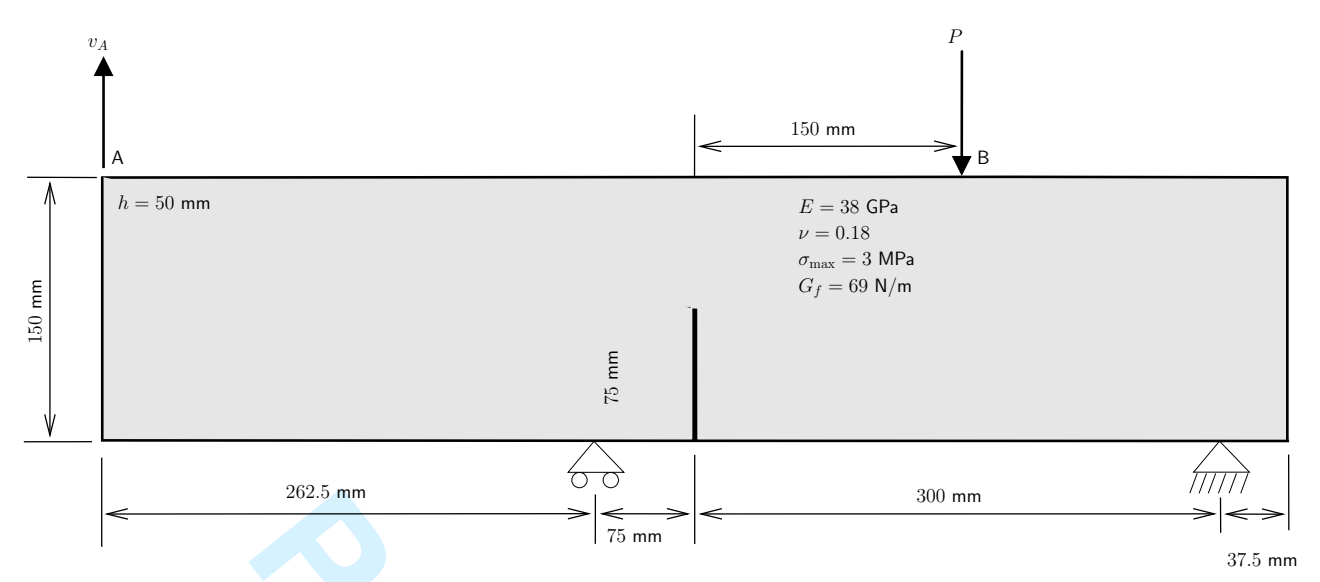

Figure 13. Relevant data for the single edge notched beam (see details in reference [37]). The mesh contains 2625 elements.

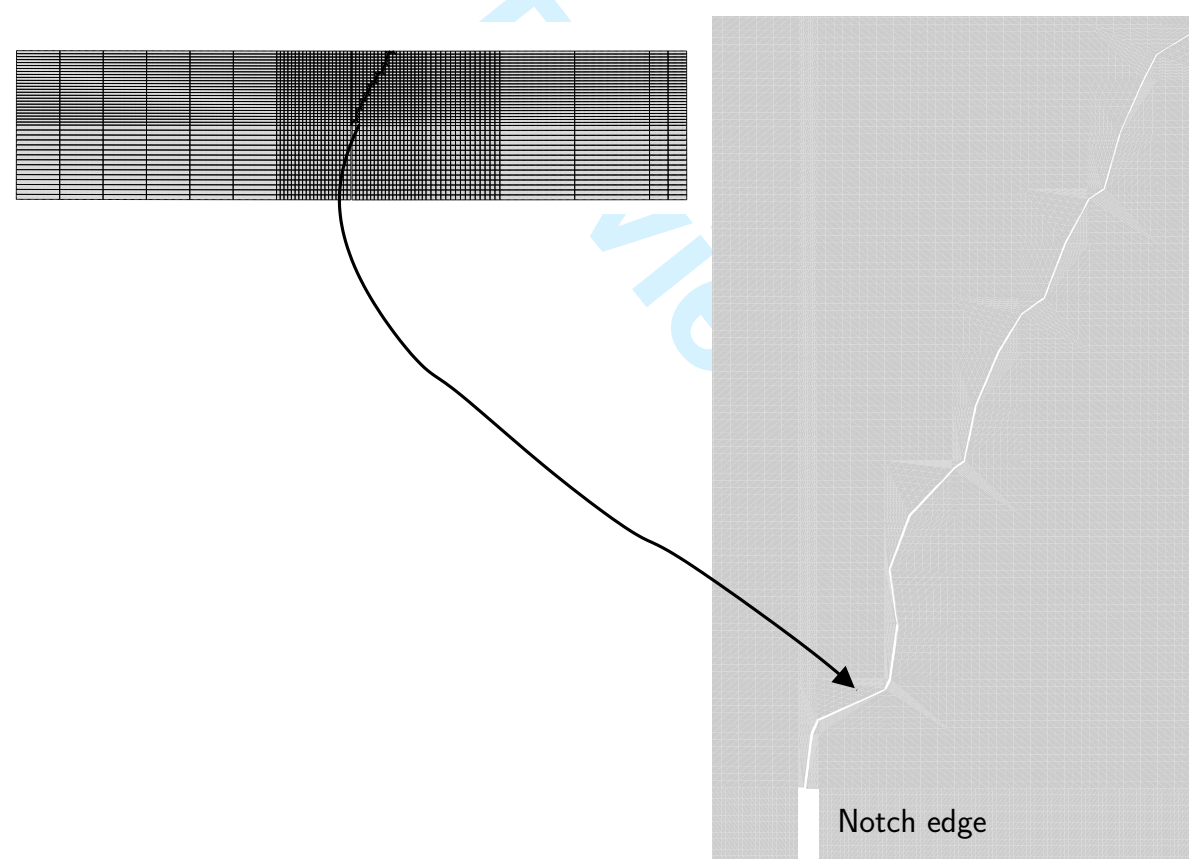

Figure 14. Crack path (over the real deformed geometry) and tip detail for the single edge notched beam. 


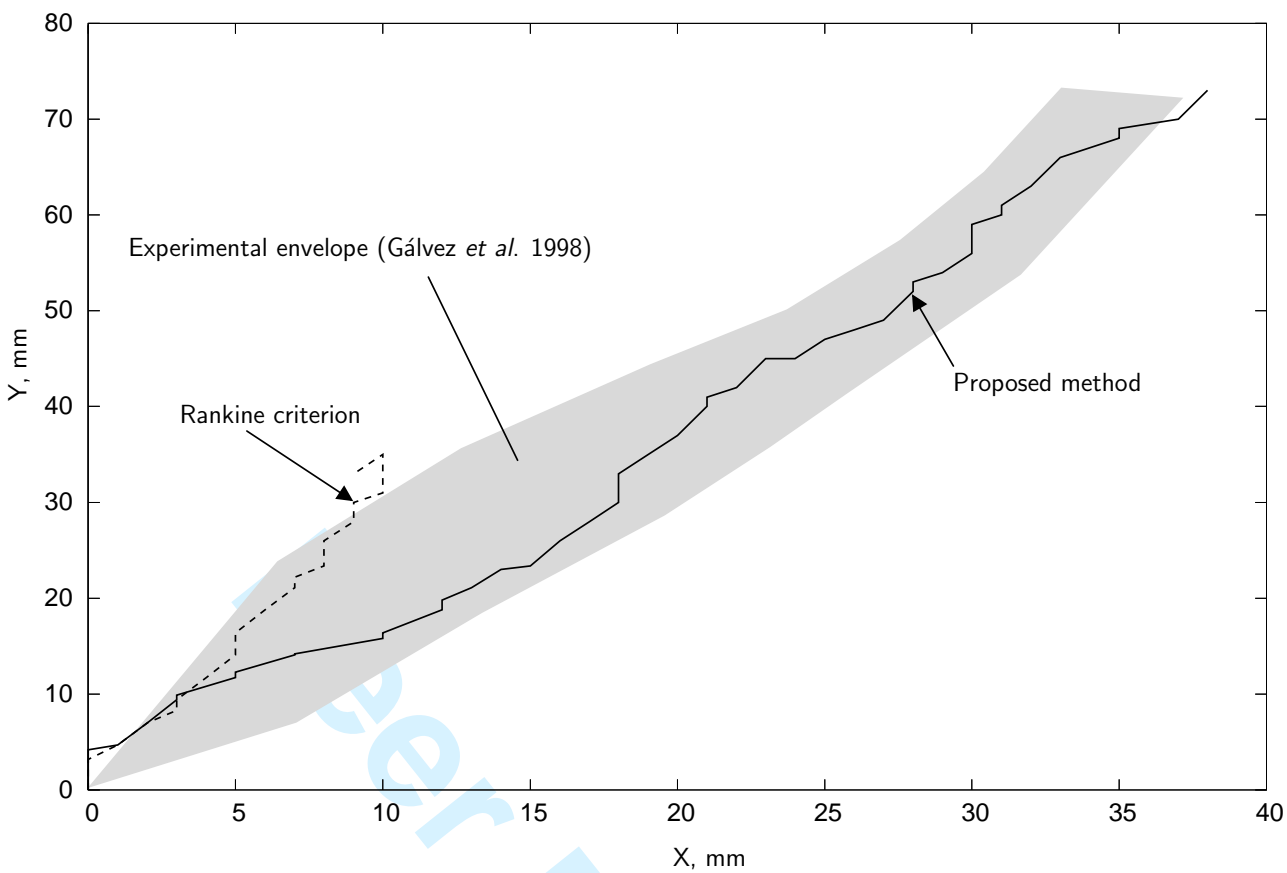

Figure 15. Crack path comparison between the proposed method and the Rankine criterion for the single edge notched beam.

Figure 19 shows the relevant ingredients of the problem. The deformed mesh with a crack is shown in figure 19. The crack path under proportional loading obtained with our method is clearly smooth.

A comparison with the experimental envelope of Gálvez et al. [39] is shown in figure 21. There is a good agreement between the experimental data and the predicted results. Note that fracture of PMMA requires large strain capabilities, which we have. 


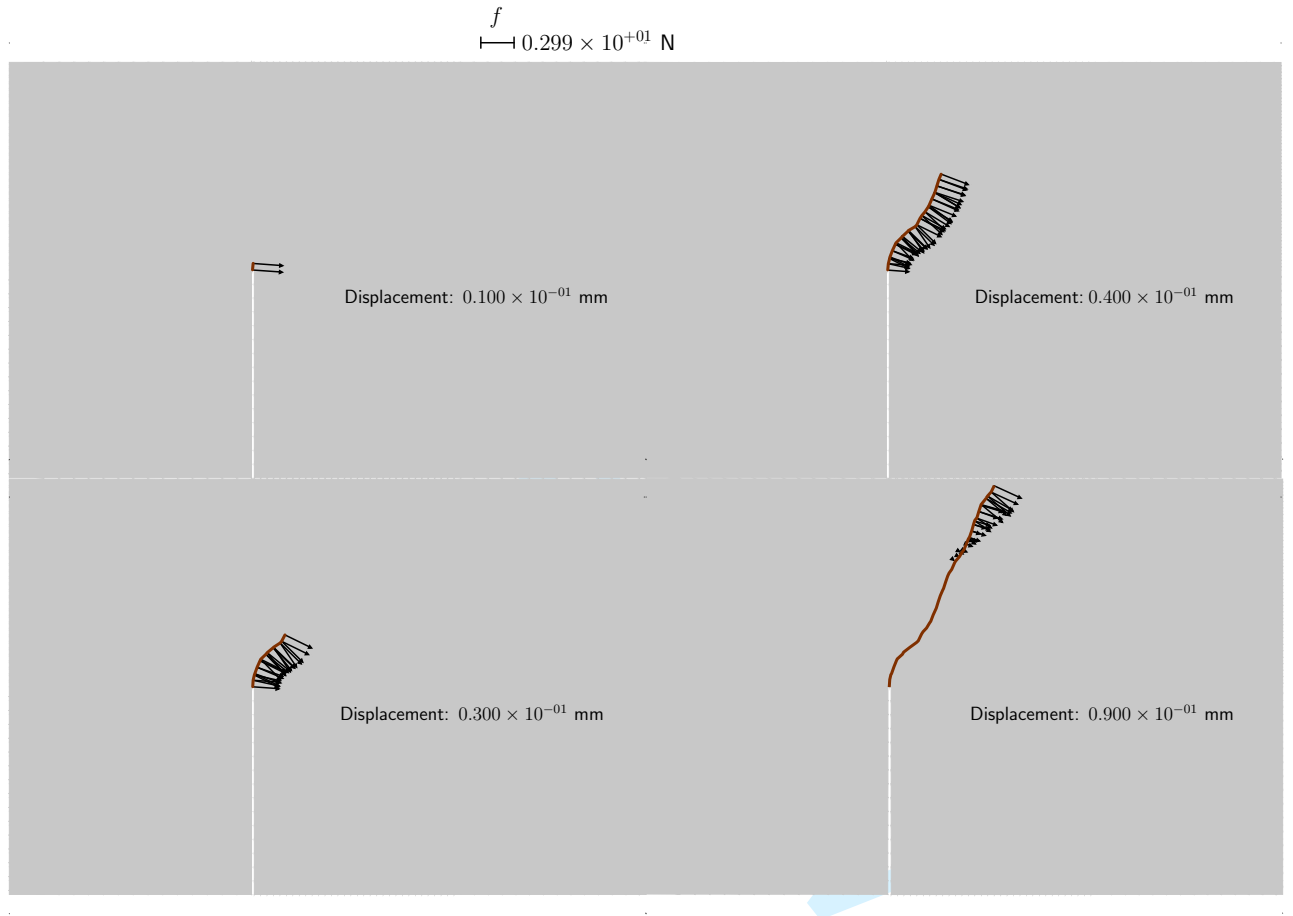

Figure 16. Sequence of time-steps with the distribution of the cohesive stress $f$. The value of the vertical displacement of point $B$ in figure 13 is shown. Note that only the relevant propagation region is depicted. 


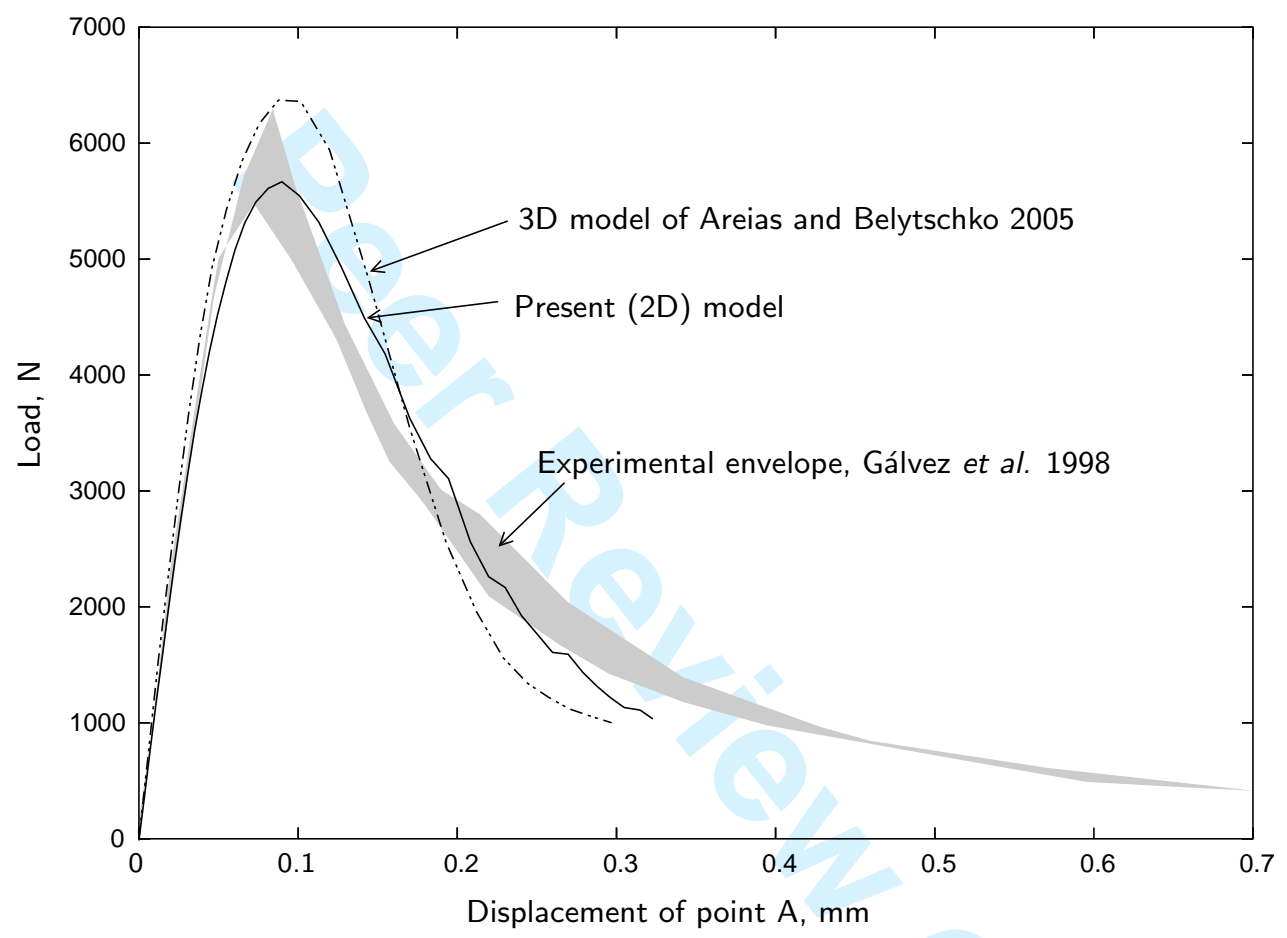

Figure 17. Single edge notched beam: load versus displacement of point $A, v_{A}$. The experimental envelope from [37] is also shown. 


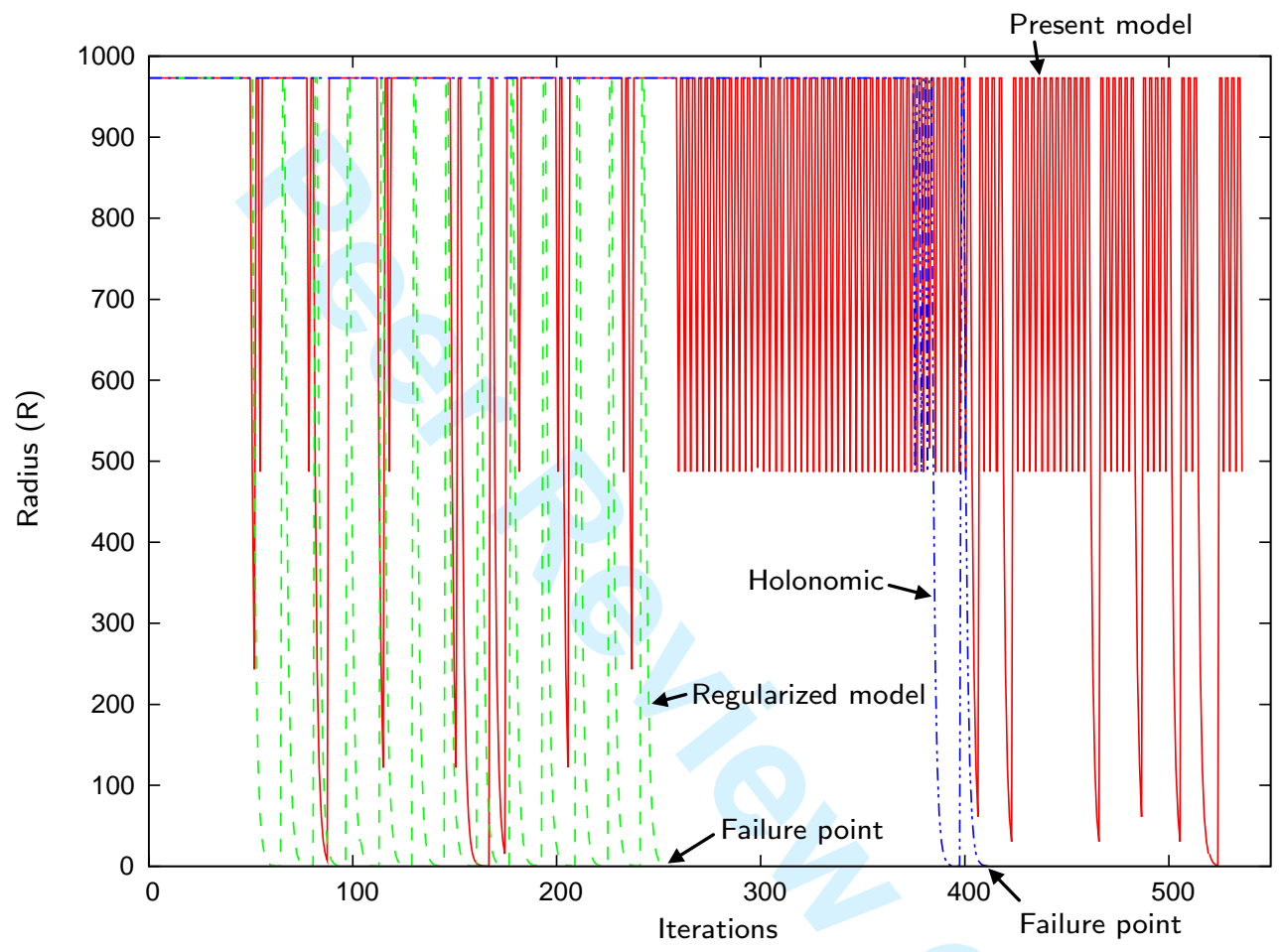

Figure 18. Single edge notched beam: trust radius as a function of the number of iterations for all time steps. For comparison, the results of a regularized model (with a penalty parameter equal to $\left.f_{\max } 1 \times 10^{3} / d_{c}\right)$ are also shown, along with the holonomic case. 


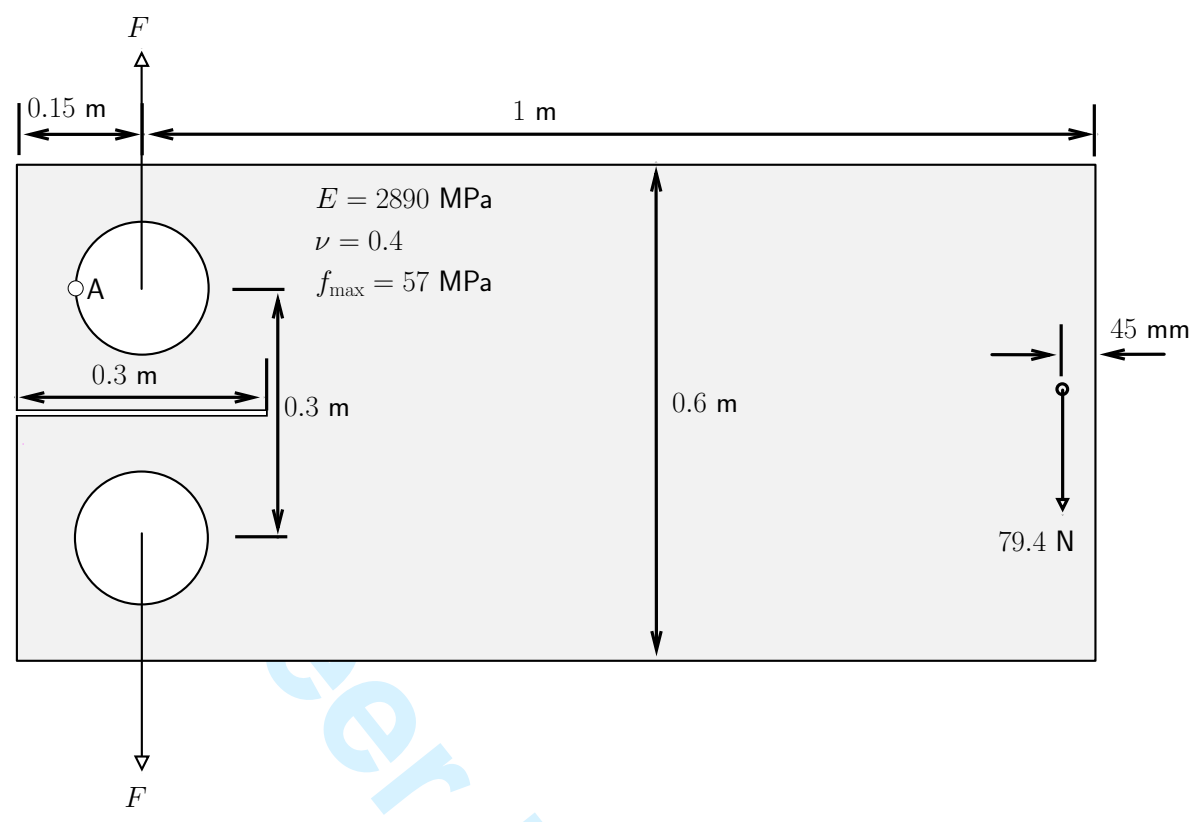

Figure 19. Geometry, boundary conditions, and relevant material properties for the double-edge notched beam.

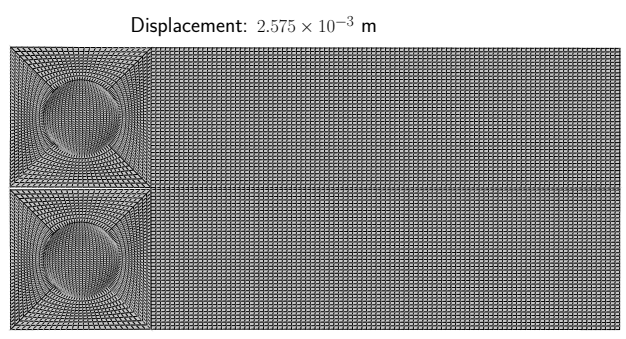

Displacement: $1.457 \times 10^{-2} \mathrm{~m}$

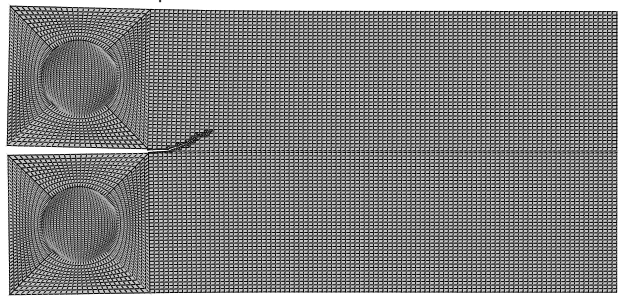

Displacement: $1.758 \times 10^{-2} \mathrm{~m}$

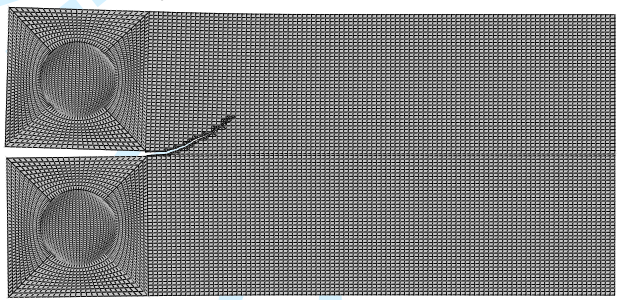

Displacement: $2.958 \times 10^{-2} \mathrm{~m}$

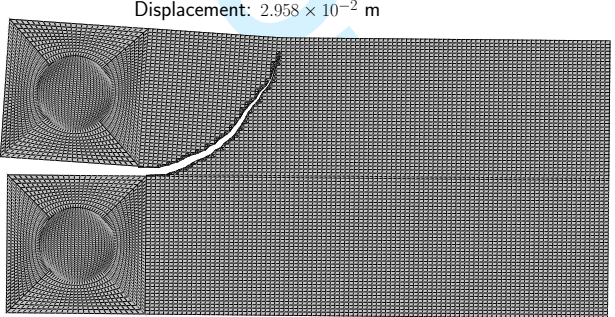

Figure 20. Sequence of deformed (coarse) meshes with explicit crack path. The displacement of point $\mathrm{A}$ is monitored.

Copyright (C) 2004 John Wiley \& Sons, Ltd.

Int. J. Numer. Meth. Engng 2004; 00:1-6 


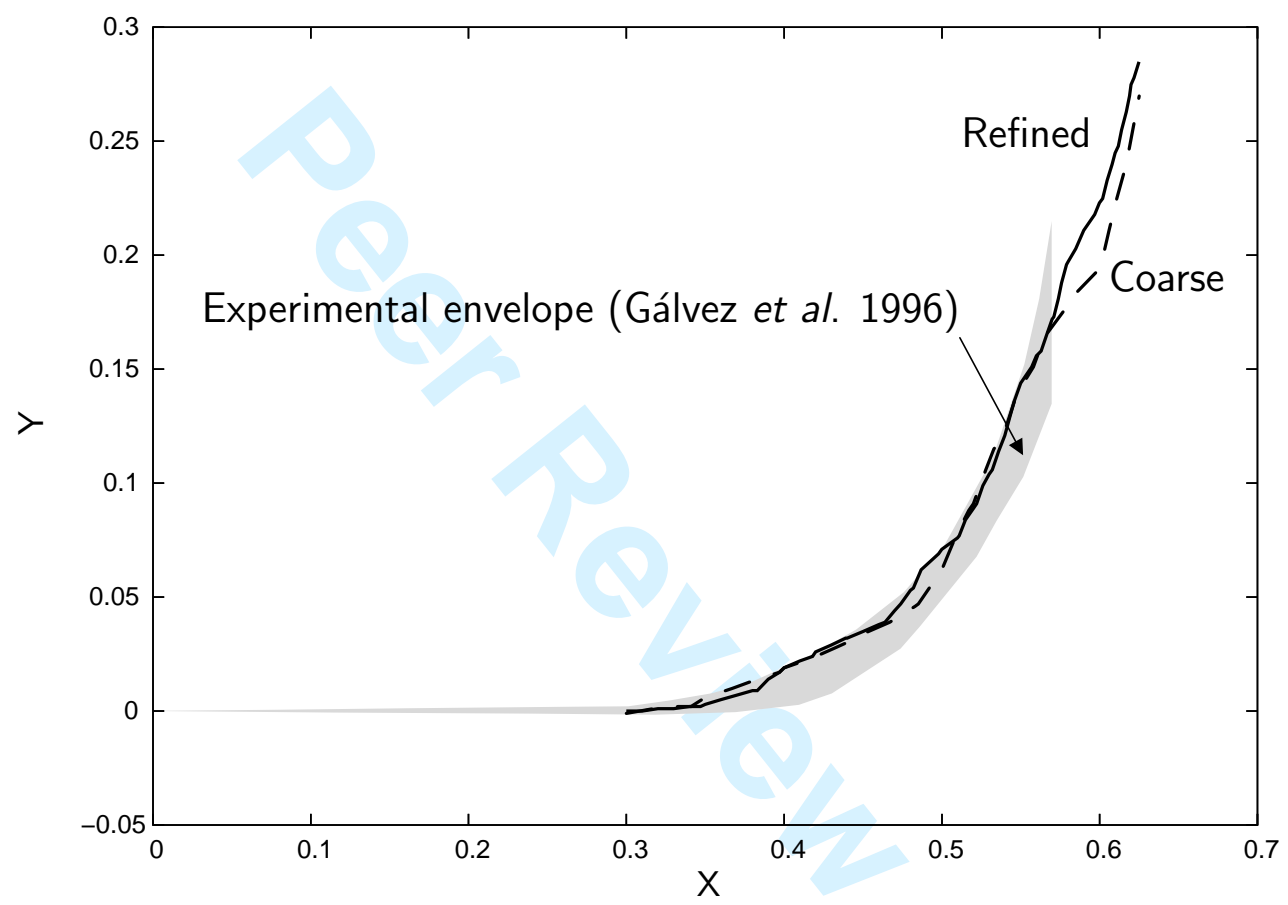

Figure 21. Experimental [39] and predicted crack paths. The coarse mesh contains 12700 elements and the refined mesh 40400 . 


\subsection{Out of plane bending}

It can be observed that less intricate methods are available to obtain some of the previous results. In the presence of out-of-plane bending, the method works works differently.

In rods, plates and shells, through-the-thickness cracks occur when at least one, but not necessarily all, points in a given cross-section satisfy a given critical condition. If not critical, remaining points should then behave as if no crack existed.

Let us consider the beam depicted in figure 22. Load-deflection results for this new situation are shown in figure 23. For $G_{c}=10000$, the evolution of the cohesive stress vectors at the boundary are shown in figure 24. It can be seen that a non-uniform distribution of stress magnitudes occurs in compression. For $v=22.0 \times 10^{-4}$ only the inner core of the clamped region is cracked. The lower edge of the clamped region is under tension and opening whereas the upper region closes in contact and adhesion because $f<f_{\max }$. It can be observed that shell kinematics are sufficient to model a wide variety of crack propagation situations.

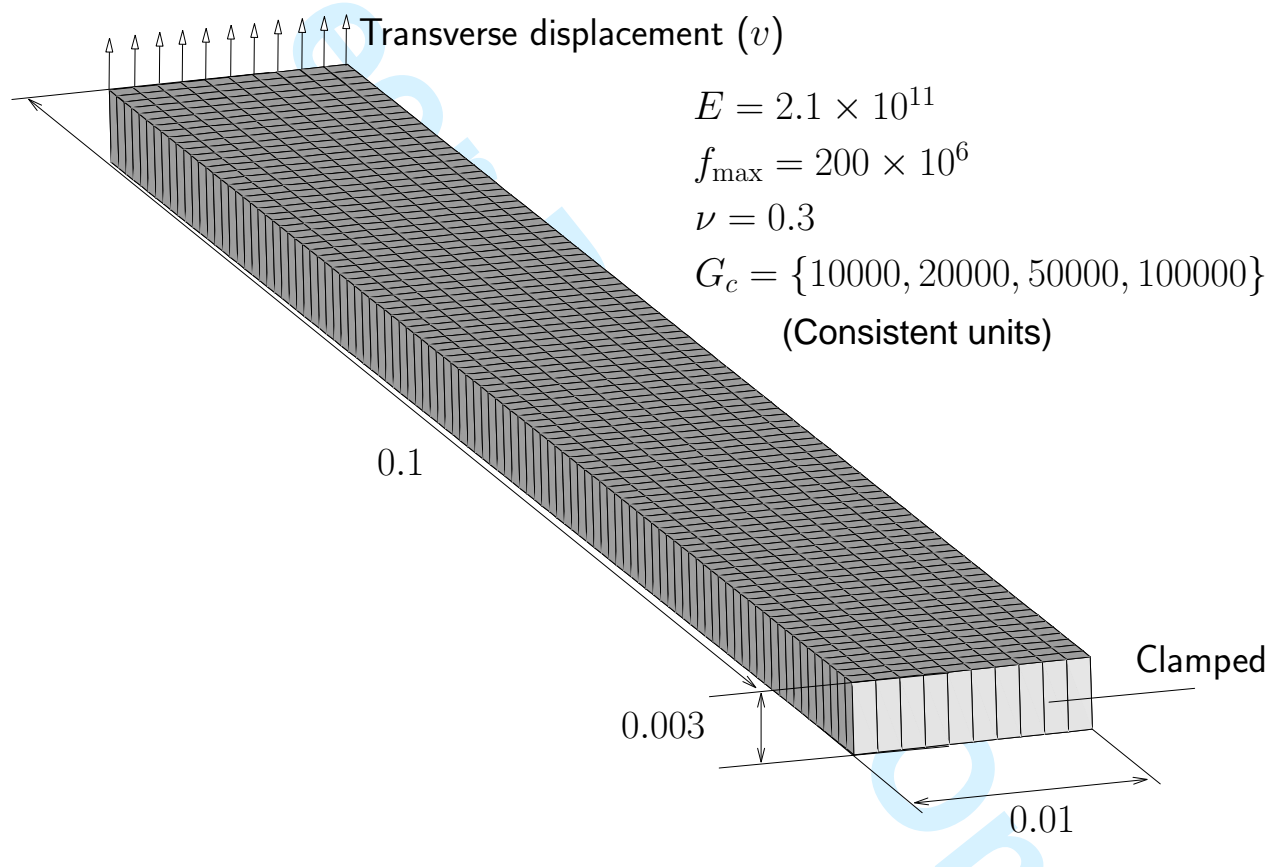

Figure 22. Out of plane bending of a beam: mesh, boundary conditions and material properties (discretization with 1000 elements). Consistent units are used.

To inspect the solution performance, a tolerance $(t o l)$-versus number of iterations $\left(n_{\mathrm{iter}}^{\max }\right)$ study is performed for $G_{c}=10000$ and using the last analysis point shown in figure 25 (it corresponds to $v=6.25 \times 10^{-3}$ ). We choose this case as it forces the crossing of two high curvature regions in loading. The relative DOF tolerance is $t o l_{t r}=1 \times 10^{-7}$. Figure 25 shows that the total number of iterations is nearly independent of tol. Therefore, the smoothed law can be made as accurate as desired.

Int. J. Numer. Meth. Engng 2004; 00:1-6 


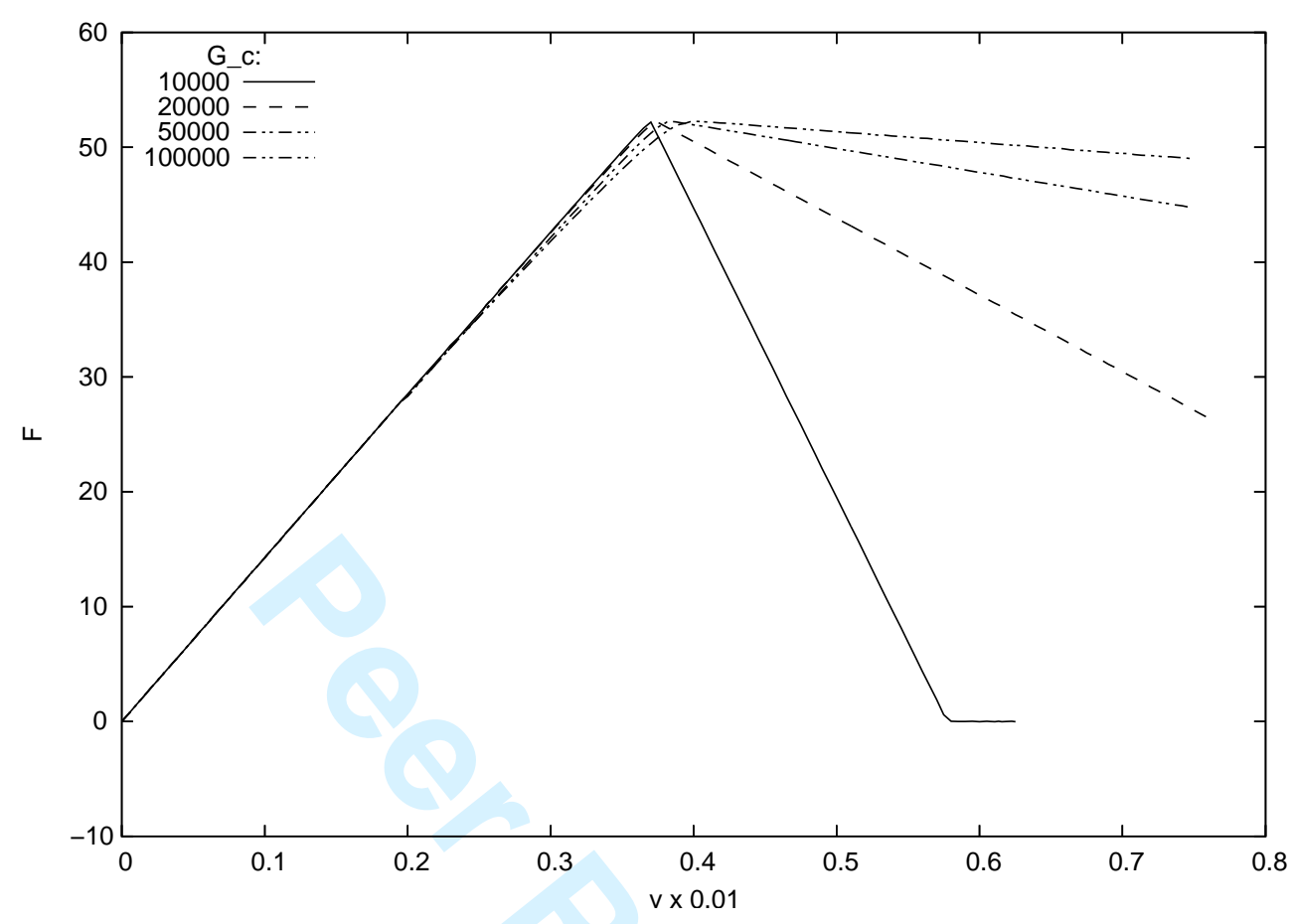

Figure 23. Load-deflection results for several values of $G_{c}$.

\subsection{Crack path in a simply supported square plate}

This example shows mode I fracture in a simply supported square plate subject to uniform, motion-dependent pressure. The purpose here is to test the crack path algorithm in mode I. The plate dimensions are $1 \times 1 \times 0.01$ consistent units, the elastic properties are $E=210 \times 10^{9}$, $\nu=0.3$ for the elasticity modulus and the Poisson coefficient, respectively. The maximum normal stress is $f_{\max }=500 \times 10^{6}$. The crack path is calculated using the COD. Two regular meshes are used: $20 \times 20$ and $100 \times 100$.

A sequence of deformed configurations is shown in figure 26. The displacement components of the mid-surface are prescribed at the boundary and therefore pressure can increase up to very high values. Due to the use of COD components, determination of the crack path requires a element-by-element approach, and this results in some irregularity, also seen before in the $2 \mathrm{D}$ example. In reality, inhomogeneity will eventually produce an analogous effect. We inhibited the "crossed" crack pattern known to occur in the simply supported case, as this would create an unnecessarily complex situation for the degrees-of-freedom in the center of the plate (usually this is solved empirically by removing the affected element). Wrinkles occur around the main crack because pressure starts to bend the crack faces.

The pressure versus mid-point displacement results are shown in figure 27 for the coarse and refined meshes. Some difference is observable, since crack paths are slightly distinct. In addition, localized bending behavior tends to increase the out-of-plane displacement at the 

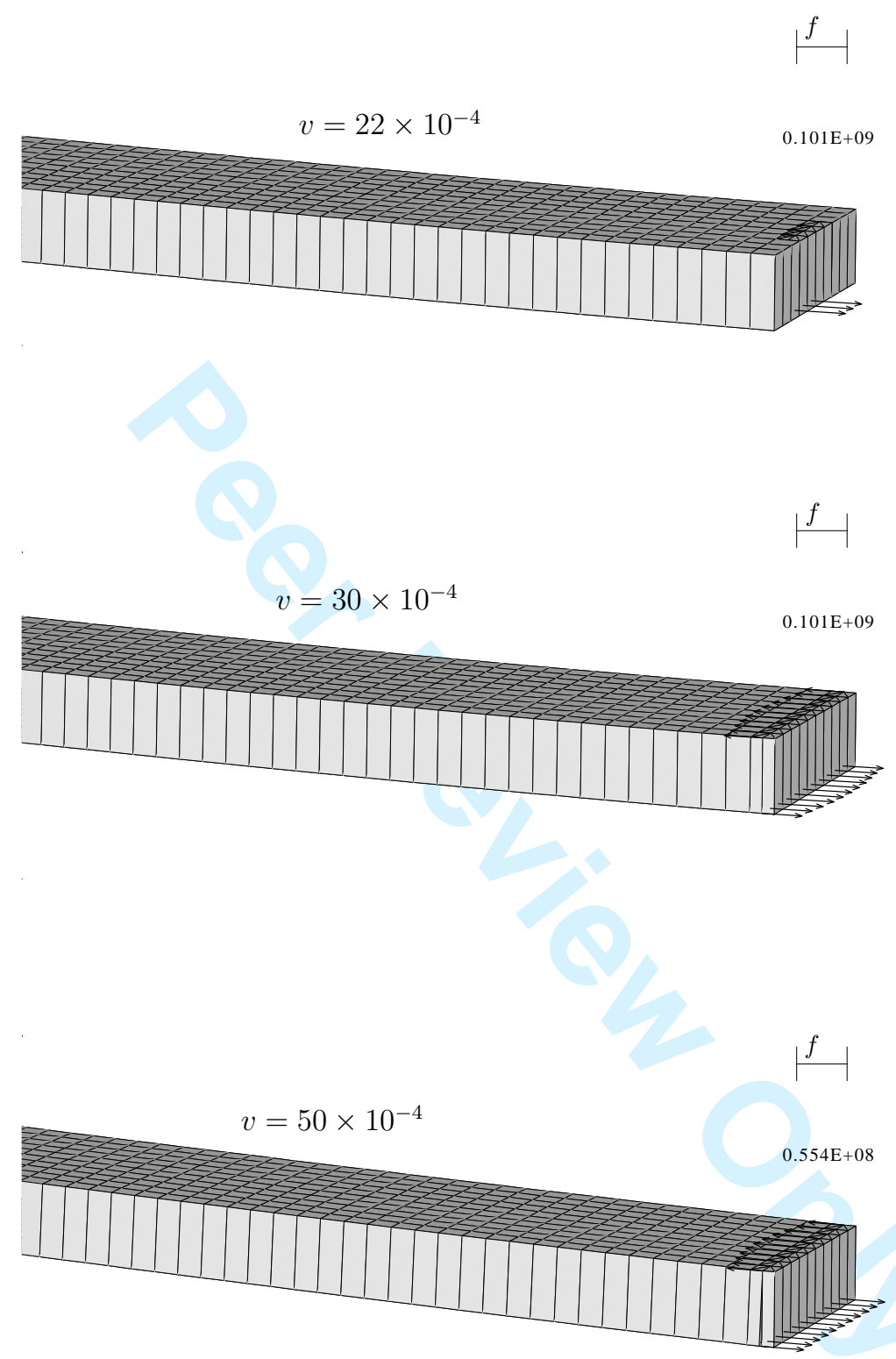

Figure 24. Evolution of cohesive stress vectors for $G_{c}=10000$ consistent units. 


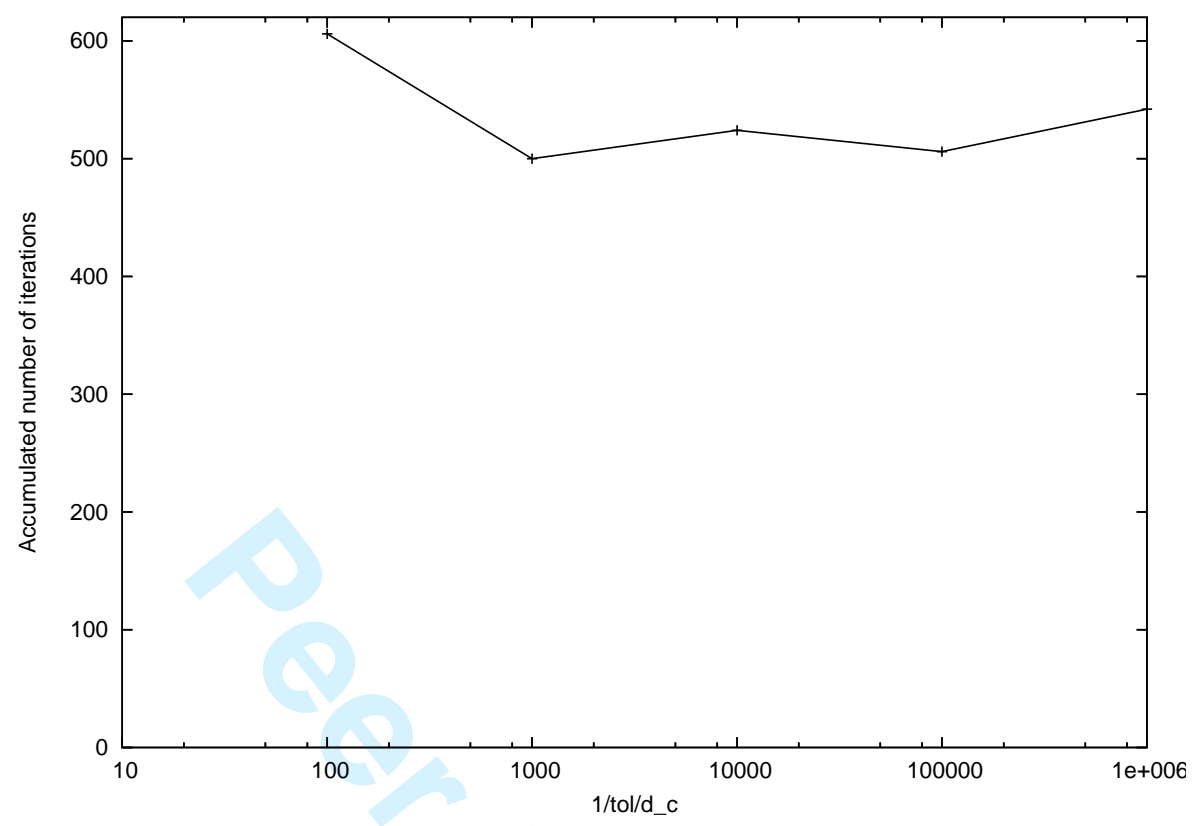

Figure 25. Results $\left(\mathrm{tol} / d_{c}\right)$ versus number of iterations $\left(n_{\mathrm{iter}}^{\max }\right)$ for $G_{c}=10000$ and $v=6.25 \times 10^{-3}$.

plate's center.

\subsection{Circular plate under pressure: mode III}

A clamped circular plate with uniform mid-surface pressure, is allowed to fracture in a circular section of the clamped boundary. Consistent units are used; the plate radius is 1 and thickness is 0.01 . The elasticity modulus is $E=210 \times 10^{9}$, Poisson coefficient is $\nu=0.3$ and the maximum shear stress is $f_{\max }=7 \times 10^{5}$. Maximum applied pressure is $p=1 \times 10^{6}$.

A small premature crack originating from an imperfection (90\% of the critical stress) is triggered in two elements in that region.

Three mesh densities (1200, 2700 and 4800 elements) and two values of fracture energy $\left(G_{c}=0\right.$ and $\left.G_{c}=50000 \mathrm{~N} / \mathrm{m}\right)$ are used for comparison. A critical value of 2 degrees in the CTOA is employed for the crack advance in the $G_{c}=0$ case. Pressure is increased in steps of 500 consistent units which were found to be sufficient to avoid artificial crack arrest.

A sequence of deformed meshes with the cohesive vectors is shown in figure 28. The evolution of the cohesive stress vectors (the product of mode III direction and the force degree of freedom) at early stages is represented in the figure 29. Relatively large openings still produce substantial stress. 

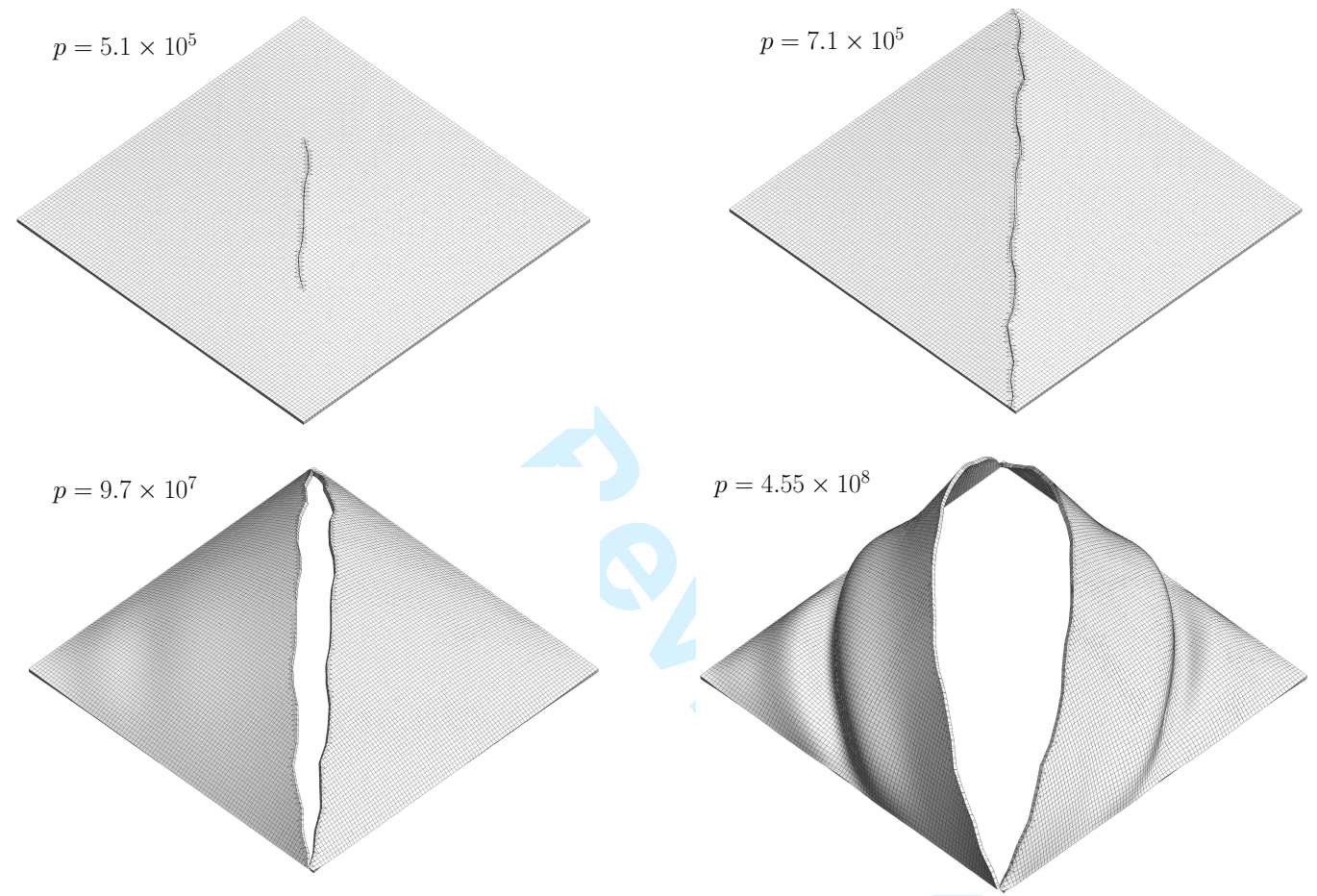

Figure 26. Square plate under pressure: sequence of deformed configurations (non-prescribed crack path). The refined mesh is shown. 


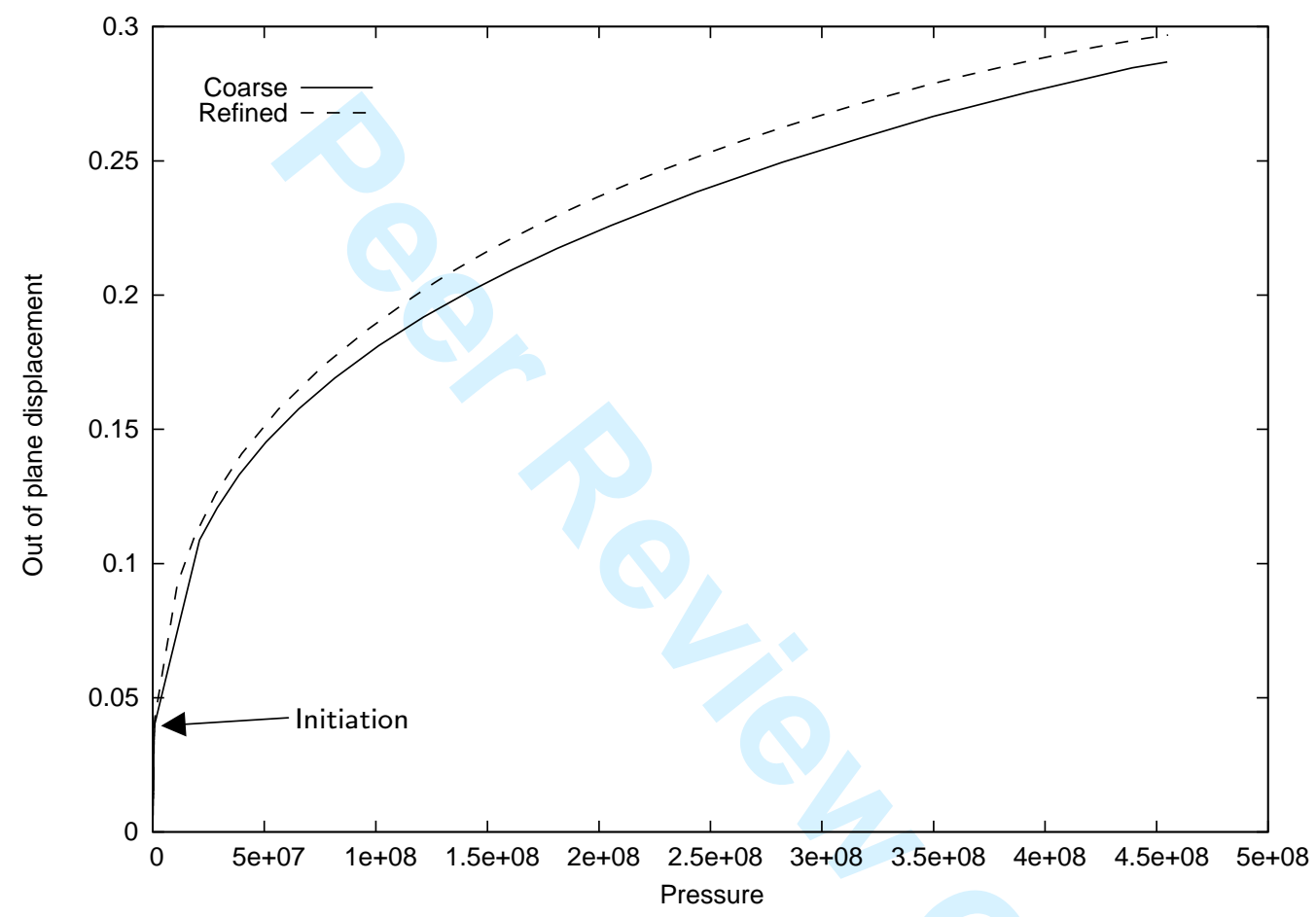

Figure 27. Square plate: pressure/mid-point displacement results. 

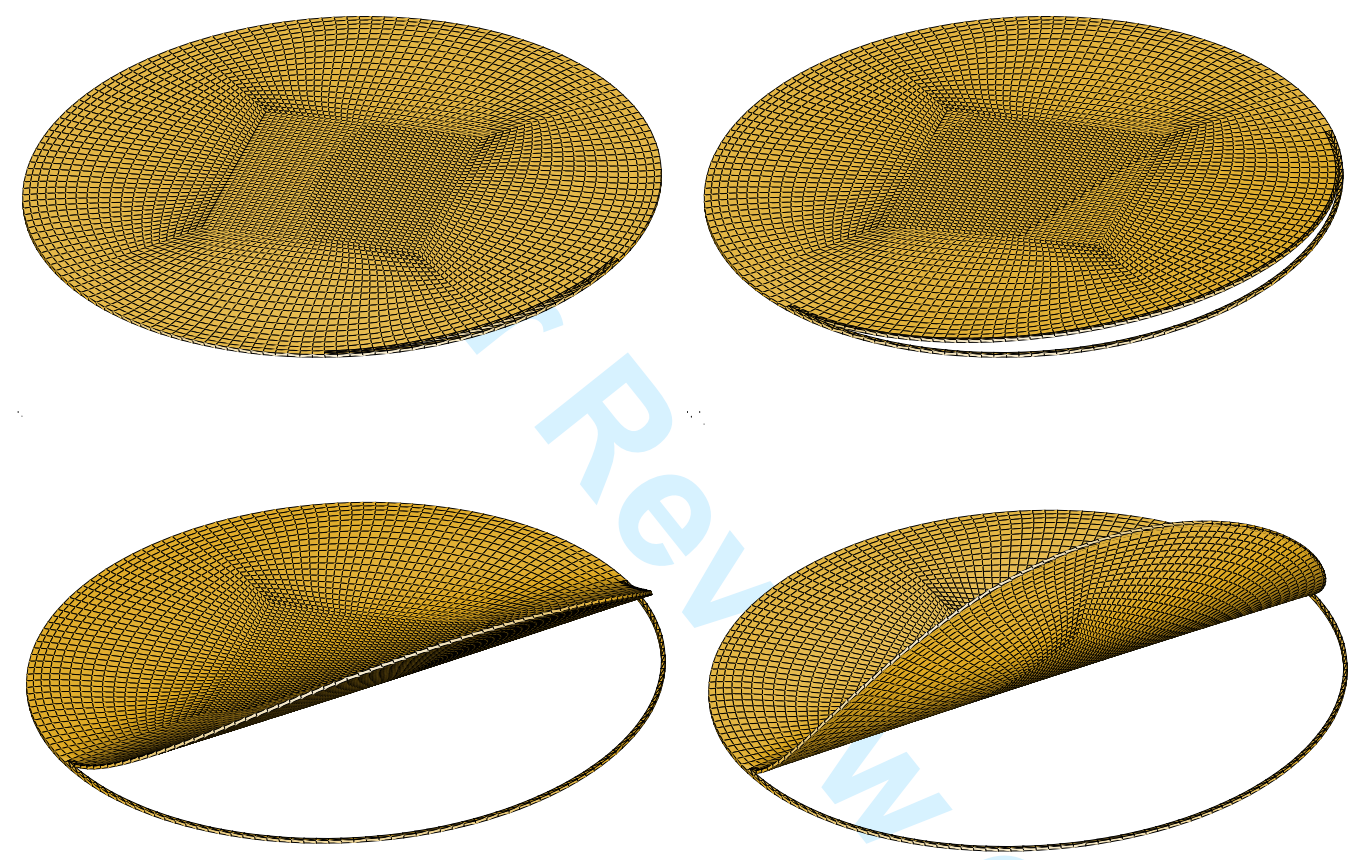

Figure 28. Circular plate under uniform pressure: Sequence of time steps. 


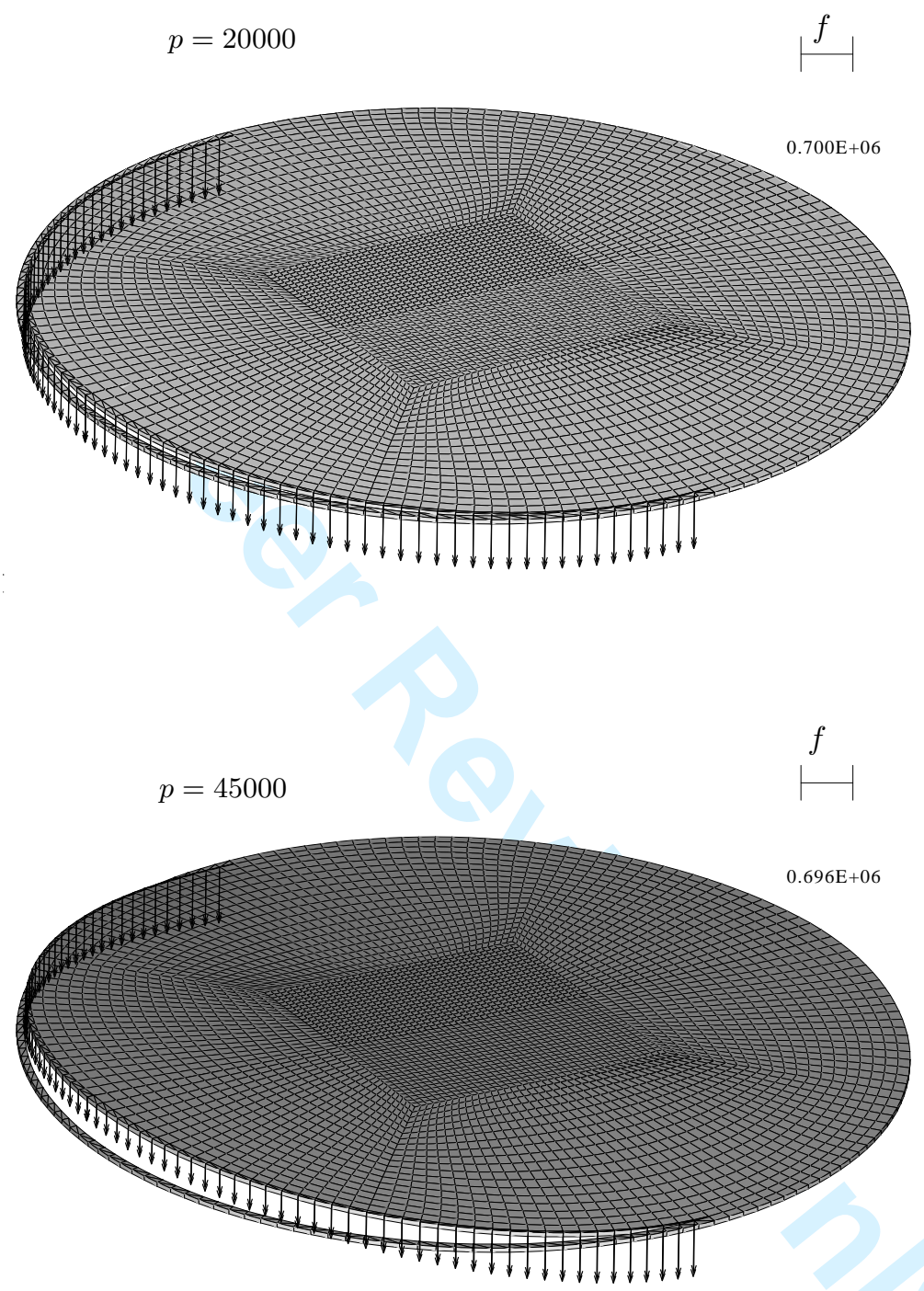

(Consistent units)

Figure 29. Circular plate under uniform pressure: Cohesive stress vectors.

The load versus out-of-plane displacement results are shown in figure 30. Robust and reasonably mesh independent results are obtained. It is worth noting that the fracture indicator has a substantial effect if $G_{c}=0$. A difference in the pressure value is indicated in figure 30 . 
The reason for this is that cohesive forces are absent and therefore a sudden jump occurs at initiation. If $G_{c} \neq 0$, there is no difference, since the cohesive forces hold the two parts together before the release. The difference can be eliminated by using a small value for $G_{c}$.

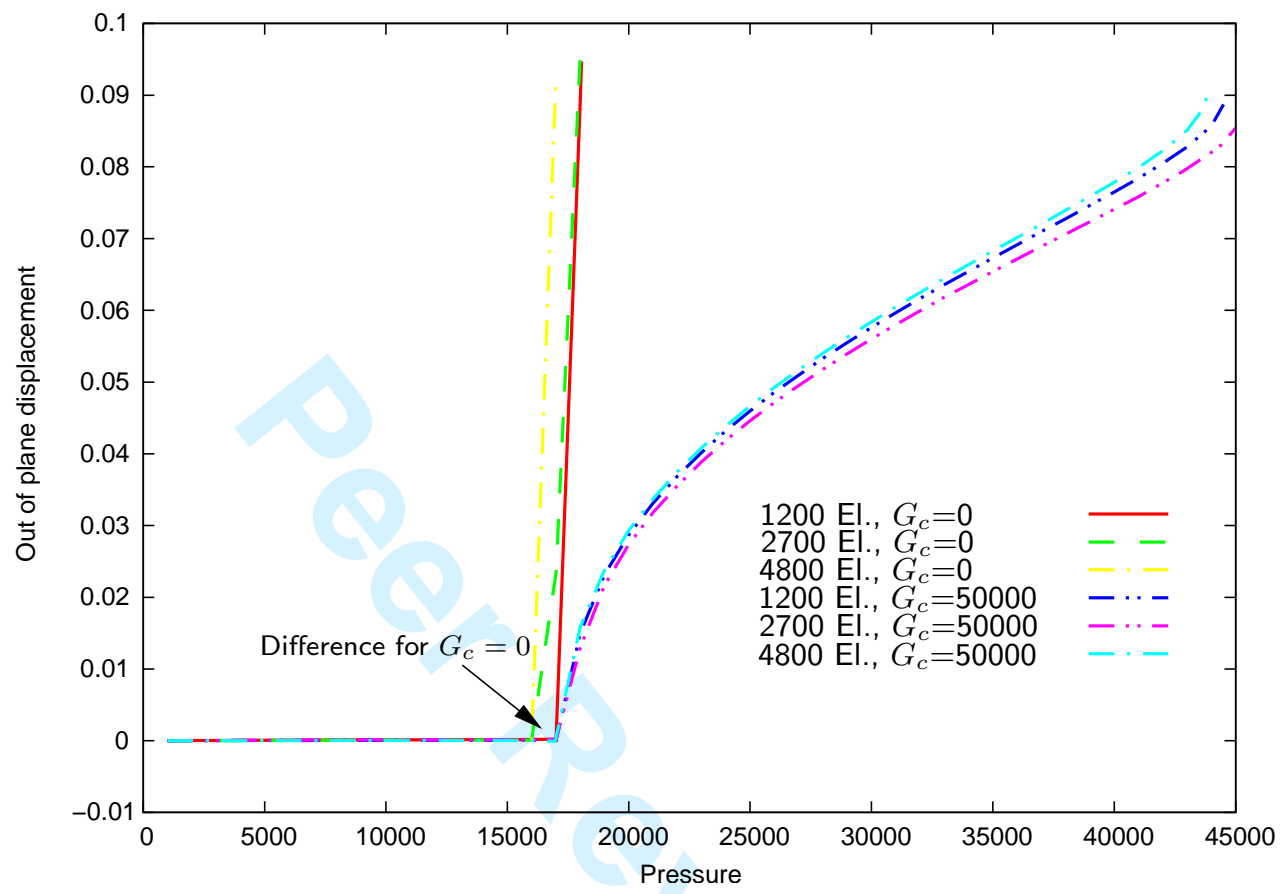

Figure 30. Circular plate under uniform pressure: Load versus out-of-plane displacement. 


\section{CONCLUSION}

The equilibrium problem with a cohesive traction-separation law was written as a complementarity problem. A method based on smooth replacement of the plus $f(x) \equiv x_{+}=$ $\max (0, x)$ and sign functions involved in the complementarity problem was introduced. This allowed a classical root-finding method to be applied to an enlarged algebraic nonlinear (and non-symmetric) system, but involving smooth functions only. Specifically, a variant of the trust region method was used in the solution of this problem.

Within this framework, loading, reloading and unloading situations were represented as accurately as desired. The method allowed the use of through-the-thickness cracks in plates for situations where not all the points along the thickness were critical at initiation. It was also verified that the crack path could be directly determined, and the method was applied to a variety of situations with high degree of success. The fracture propagation part of the code is now very robust.

\section{ACKNOWLEDGEMENTS}

The authors would like to thank Professor T. Belytschko for his advice and also one of the reviewers which suggested numerous improvements.

\section{REFERENCES}

1. G.I. Barenblatt. The mathematical theory of equilibrium of cracks in brittle fracture. Advances in Applied Mechanics, 7:55-129, 1962.

2. J. Planas and M. Elices. Nonlinear fracture of cohesive materials. International Journal of Fracture, 51:139-157, 1991.

3. Z.P. Bažant and J. Planas. Fracture and size effect in concrete and other quasibrittle materials. CRC Press, 1998.

4. M. Elices, G.V. Guinea, J. Gómez, and J. Planas. The cohesive zone model: advantages, limitations and challenges. Engineering Fracture Mechanics, 69:137-163, 2002.

5. J. Planas, M. Elices, G.V. Guinea, D.A. Gómez, Cendón, and I. Arbilla. Generalizations and specializations of cohesive crack models. Engineering Fracture Mechanics, 70:1759-1776, 1996.

6. G. Alfano and M.A. Crisfield. Finite element interface models for the delamination analysis of laminated composites: mechanical and computational issues. International Journal for Numerical Methods in Engineering, 50:1701-1736, 2001.

7. D.V. Kubair and P.H. Geubelle. Comparative analysis of extrinsic and intrinsic cohesive models of dynamic fracture. International Journal of Solids and Structures, 40:3853-3868, 2003.

8. C.-H. Sam, K.D. Papoulia, and S.A. Vavasis. Obtaining initially rigid cohesive finite element models that are temporally convergent. Engineering Fracture Mechanics, 72:2247-2267, 2005.

9. K.D. Papoulia, C.-H. Sam, and S.A. Vavasis. Time continuity in cohesive finite element modeling. International Journal for Numerical Methods in Engineering, 58:679-701, 2003.

10. R.I. Leine and H. Nijmeijer. Dynamics and bifurcations of non-smooth mechanical systems. Springer, 2004.

11. G. Bolzon, G. Maier, and F. Tin-Loi. On multiplicity of solutions in quasi-brittle fracture computations. Computational Mechanics, 19:511-516, 1997.

12. T. Belytschko. Private communication. 2003

13. A.E. Huespe, J. Oliver, M.D.G. Pulido, S. Blanco, and D. Linero. On the fracture models determined by the continuum-strong discontinuity approach. International Journal of Fracture, 137:211-229, 2006.

14. P.M.A. Areias, J.M.A. César de Sá, C.A. Conceição António, J.A.S.A.O. Carneiro, and V.M.P. Teixeira. Strong displacement discontinuities and Lagrange multipliers in the analysis of finite displacement fracture problems. Computational Mechanics, 35:54-71, 2004. 
15. P. M. A. Areias, J. H. Song, and T. Belytschko. Analysis of fracture in thin shells by overlapping paired elements. Computer Methods in Applied Mechanics and Engineering, 195(41-43):5343-5360, 2006.

16. J.-S. Pang. Newton's method for B-differentiable equations. Mathematics of Operations Research, 15:311341, 1990.

17. C. Chen and O.L. Mangasarian. A class of smoothing functions for nonlinear and mixed complementarity problems. Computational Optimization and Applications, 5:97-138, 1996.

18. B. Chen and P.T. Harker. Smooth approximations to nonlinear complementarity problems. SIAM Journal of Optimization, 7(2):403-420, 1997.

19. P. M. A. Areias, J. H. Song, and T. Belytschko. Finite-strain quadrilateral shell element based on discrete kirchhoff-love constraints. International Journal for Numerical Methods in Engineering, 64(9):1166-1206, 2005.

20. N. Moës, J. Dolbow, and T. Belytschko. A finite element method for crack growth without remeshing. International Journal for Numerical Methods in Engineering, 46:131-150, 1999.

21. T. Belytschko and T. Black. Elastic crack growth in finite elements with minimal remeshing. International Journal for Numerical Methods in Engineering, 45:601-620, 1999

22. T. Belytschko, N. Moës, S. Usui, and C. Parimi. Arbitrary discontinuities in finite elements. International Journal for Numerical Methods in Engineering, 50:993-1013, 2001.

23. G.I. Barenblatt. On some problems of the theory of elasticity arising in investigating the mechanism of hydraulic fracture of an oil-bearing stratum. Prikladnaya Matematika i Mekhanika, 20:475-486, 1956. (In Russian).

24. G.I. Barenblatt. On the equilibrium cracks due to brittle fracture. Doklady AN SSSR, 127:47-50, 1959. (In Russian).

25. J.N. Goodier. Mathematical theory of equilibrium cracks, volume II of Fracture, chapter I, pages 1-66. Academic Press, 1969.

26. N. Moës and T. Belytschko. Extended finite element method for cohesive crack growth. Engineering Fracture Mechanics, 69:813-833, 2002.

27. A.L. Eterovic and K.J. Bathe. On the treatment of inequality constraints arising from contact conditions in finite element analysis. Computers and Structures, 40(2):203-209, 1991.

28. X.-P. Xu and A. Needleman. Numerical simulations of fast crack growth in brittle solids. Journal of the Mechanics and Physics of Solids, 42(9):1397-1434, 1994.

29. M.L. Falk, A. Needleman, and J.R. Rice. A critical evaluation of dynamic fracture simulations using cohesive surfaces. Journal de Physique IV, 11:43-52, 2001.

30. C. Lanczos. The variational principles of mechanics. Dover publications, fourth edition, 1986. Reprint from University of Toronto Press, 1970.

31. F. Tin-Loi and P. Tseng. Efficient computation of multiple solutions in quasibrittle fracture analysis. Computer Methods in Applied Mechanics and Engineering, 192:1377-1388, 2003.

32. F. Tin-Loi and N.S. Que. Nonlinear programming approaches for an inverse problem in quasibrittle fracture. International Journal of Mechanical Sciences, 44:843-858, 2002.

33. M.F. Kanninen and C.H. Popelar. Advanced Fracture Mechanics. The Oxford Engineering Science Series. Oxford University Press, 1985.

34. J.M.B. Walmag and E.J.M. Delhez. A note on trust-region radius update. SIAM Journal of Optimization, 16(2):548-562, 2005.

35. B. Chen and P.T. Harker. A non-interior-point continuation method for linear complementarity problems. SIAM Journal on Matrix Analysis and Applications, 14:1168-1190, 1993.

36. M.A. Sutton, X. Deng, F. Ma, J.C. Newman Jr, and M. James. Development and application of a crack tip opening displacement-based mixed mode fracture criterion. International Journal of Solids and Structures, $37: 3591-3618,2000$.

37. J.C. Gálvez, M. Elices, G.V. Guinea, and J. Planas. Mixed mode fracture of concrete under proportional and nonproportional loading. International Journal of Fracture, 94:267-284, 1998.

38. P.M.A. Areias and T. Belytschko. Analysis of three-dimensional crack initiation and propagation using the extended finite element method. International Journal for Numerical Methods in Engineering, 63:760$788,2005$.

39. J. Gálvez, M. Elices, G.V. Guinea, and J. Planas. Crack trajectories under mixed mode and nonproportional loading. International Journal of Fracture, 81:171-193, 1996.

40. M. Shen and M.-H. H. Shen. Direction of crack extension under general loading. International Journal of Fracture, 70:51-58, 1995. 